\title{
Subspace-Based Damage Detection under Changes in the Ambient Excitation Statistics ${ }^{\text {th }}$
}

\author{
Michael Döhler ${ }^{\mathrm{a}, *}$, Laurent Mevel ${ }^{\mathrm{b}}$, Falk Hille ${ }^{\mathrm{c}}$ \\ ${ }^{a}$ Northeastern University, Department of Civil and Environmental Engineering, Center for Digital Signal Processing, 360 Huntington Avenue, \\ Boston, MA 02115, USA \\ ${ }^{b}$ Inria, Centre Rennes - Bretagne Atlantique, Campus de Beaulieu, 35042 Rennes, France \\ ${ }^{c}$ BAM Federal Institute for Materials Research and Testing, Safety of Structures Department, 12200 Berlin, Germany
}

\begin{abstract}
In the last ten years, monitoring the integrity of the civil infrastructure has been an active research topic, including in connected areas as automatic control. It is common practice to perform damage detection by detecting changes in the modal parameters between a reference state and the current (possibly damaged) state from measured vibration data. Subspace methods enjoy some popularity in structural engineering, where large model orders have to be considered. In the context of detecting changes in the structural properties and the modal parameters linked to them, a subspacebased fault detection residual has been recently proposed and applied successfully, where the estimation of the modal parameters in the possibly damaged state is avoided. However, most works assume that the unmeasured ambient excitation properties during measurements of the structure in the reference and possibly damaged condition stay constant, which is hardly satisfied by any application. This paper addresses the problem of robustness of such fault detection methods. It is explained why current algorithms from literature fail when the excitation covariance changes and how they can be modified. Then, an efficient and fast subspace-based damage detection test is derived that is robust to changes in the excitation covariance but also to numerical instabilities that can arise easily in the computations. Three numerical applications show the efficiency of the new approach to better detect and separate different levels of damage even using a relatively low sample length.
\end{abstract}

Keywords: Damage detection, Structural vibration monitoring, Ambient excitation, Subspace methods, Hypothesis testing

\section{Introduction}

The fault detection problem is related to the detection and diagnosis of changes in the eigenstructure of a linear dynamical system in many applications. An important example is structural vibration monitoring, where damages of civil, mechanical or aeronautical structures lead to a change in the eigenstructure of the underlying mechanical system and thus in the modal parameters (natural frequencies, damping ratios and mode shapes). The excitation of these systems is ambient and mostly unmeasured. Vibration-based damage detection methods have been developed extensively in the last 30 years. In [2], an introduction to vibration-based damage identification is given. An overview of damage identification methods and strategies can be found in [3-5].

Often, damage detection is done by estimating the modal parameters of a structure in a possibly damaged state and comparing them to estimates from a reference condition, e.g. by using control charts [6-8]. In this context, especially the natural frequencies are used for a comparison, as they can be reliably identified. However, the automated estimation of modal parameters from a system identification method and matching them from measurements of different

\footnotetext{
${ }^{2}$ A preliminary version of this paper was presented at the 18th IFAC World Congress, August 28 - September 2, 2011, Milan, Italy [1].

${ }^{*}$ Corresponding author. Present address: BAM Federal Institute for Materials Research and Testing, Safety of Structures Department, 12200 Berlin, Germany. E-Mail: michael.doehler@bam.de, Tel. +49 8104 1701, Fax +49 81041727.

Email addresses: laurent.mevel@inria.fr (Laurent Mevel), falk.hille@bam.de (Falk Hille)
} 


\begin{tabular}{|llll|}
\hline \multicolumn{2}{l}{ Nomenclature } & & \\
& & & \\
$\mathbb{R}, \mathbb{C}$ & sets of real and complex numbers & $R_{i}$ & output covariance at lag $i$ \\
$\mathbf{E}$ & expectation of a random variable & $\mathcal{H}_{p+1, q}$ & Hankel matrix of output covariances $R_{i}$ \\
$\mathcal{N}(m, \Sigma)$ & multivariate Gaussian distribution & $O_{p+1}, C_{q}$ & observability, controllability matrix \\
$\otimes$ & Kronecker product & $\theta_{0}, \theta$ & system parameter in reference and current state \\
vec & column stacking vectorization operator & $\delta \theta$ & change in system parameter \\
$\dagger$ & Moore-Penrose pseudoinverse & $\mathbf{H}_{0}, \mathbf{H}_{1}$ & null and alternative hypotheses \\
$\widehat{(\cdot)}$ & estimate of a quantity & $S\left(\theta_{0}\right)$ & left null space of $O_{p+1}$ in reference state \\
$A, C$ & system matrices & $U_{1}$ & matrix of principal singular vectors of $\mathcal{H}_{p+1, q}$ \\
$x_{k}, y_{k}$ & states and outputs of system & $\zeta_{N}, \xi_{N}$ & residual vectors computed on $N$ data points \\
$v_{k}, w_{k}$ & state and output noise & $\mathcal{J}_{\zeta}, \mathcal{J}_{\xi}$ & asymptotic sensitivity of residual vector \\
$Q$ & excitation covariance, $\operatorname{cov}\left(v_{k}\right)$ & $\Sigma_{\zeta}, \Sigma_{\xi}$ & asymptotic covariance of residual vector \\
$\widetilde{Q}$ & excitation covariance in tested state & $\chi_{N}^{2}, \gamma_{N}^{2}$ & asymptotically $\chi^{2}$-distributed test variables \\
$Q, \mathcal{R}$ & matrices in QR decomposition & $(\cdot)$ & variable for non-parametric test variant \\
\hline
\end{tabular}

states of the structure for their comparison might require an extensive preprocessing step. Other methods avoid the system identification step in the possibly damaged state and use e.g. outlier analysis for damage detection [9], Kalman filter innovations $[10,11]$ or other data-driven features that are sensitive to changes in the modal parameters. The methods [12-14] considered in this paper compare a model obtained in the reference state to data from the possibly damaged state using a subspace-based residual function and a $\chi^{2}$-test built on it for a hypothesis test, without actually estimating the modal parameters in the tested, possibly damaged states. This approach provides a complete statistical framework for analyzing a damage residual for both reference and possibly damaged structures. The asymptotic probability distribution of the considered damage residual for both structural states is given and a statistical hypothesis test to detect damage in the structure has been proposed.

While the modal parameters of a structure are not afflicted by a change in the ambient excitation statistics, damage detection tests that avoid the system identification step and use directly the measured vibration data are possibly perturbed by changes in the excitation covariance. We assume stationary excitation during the measurement of one data set, while the excitation covariance may change between measurements. The subspace-based damage detection algorithm [12] takes into account the statistical properties of the ambient excitation in its formulation, but it is shown that it is not robust to a change in the excitation covariance. In this paper, it is shown how this test can be corrected easily by recomputing the residual covariance for every tested data set at the expense of an increase in computational burden and loss of accuracy.

In parallel, a similar subspace-based damage residual that is robust to a changing excitation covariance has been proposed in [15]. Detecting a change in the structural properties by means of such a residual is possible by a hypothesis testing procedure involving the norm of the residual pondered by a precise estimate of its covariance as explained in [12]. In this paper, the statistical framework of [12] is extended to the robust residual. The statistical properties of the robust residual are analyzed and the corresponding hypothesis test is derived. A fast and numerically stable computation of the residual covariance and the damage detection test is proposed. The efficiency of this approach and its robustness to changes in the excitation covariance are demonstrated on three numerical examples and compared to the damage detection tests from [12-14].

This paper is organized as follows. After presenting the basic principles of statistical subspace-based fault detection from [12,13] in Section 2, the impact of a changing ambient excitation covariance between measurements on the damage detection test is discussed in Section 3. A damage detection test that is robust to a changing excitation covariance is proposed in Section 4, where a robust residual function and its corresponding hypothesis test are derived. In Section 5, simplified non-parametric versions of the presented damage detection tests are derived. In Section 6, a fast and numerically robust computation of the residual covariance and the damage detection test is proposed and in Section 7 an algorithmic summary of the new robust damage detection test is given. In Section 8, numerical results of 
the proposed algorithms are presented.

\section{Background of statistical subspace-based fault detection}

In this section, the basic concepts of stochastic subspace-based fault detection from $[12,13]$ are presented.

\subsection{Models and parameters}

The use of the state-space representation for output-only vibration-based structural monitoring is well-established, which corresponds to monitoring the eigenstructure of the discrete time model

$$
\left\{\begin{aligned}
x_{k+1} & =A x_{k}+v_{k} \\
y_{k} & =C x_{k}+w_{k}
\end{aligned}\right.
$$

with the states $x_{k} \in \mathbb{R}^{n}$, the outputs $y_{k} \in \mathbb{R}^{r}$, the state transition matrix $A \in \mathbb{R}^{n \times n}$ and the observation matrix $C \in \mathbb{R}^{r \times n}$, where $r$ is the number of sensors and $n$ is the system order. The excitation $v_{k}$ is an unmeasured Gaussian white noise sequence with zero mean and constant covariance matrix $Q: \mathbf{E}\left(v_{k} v_{k^{\prime}}^{T}\right) \stackrel{\text { def }}{=} Q \delta\left(k-k^{\prime}\right)$, where $\mathbf{E}(\cdot)$ denotes the expectation operator, and $w_{k}$ is the measurement noise.

The collection of eigenvalues and mode shapes $(\lambda, \varphi)$ comprising the eigenstructure of system (1) results from

$$
\operatorname{det}\left(A-\lambda_{i} I\right)=0, \quad A \phi_{i}=\lambda_{i} \phi_{i}, \quad \varphi_{i}=C \phi_{i},
$$

where $\lambda_{i}$ and $\phi_{i}$ are the eigenvalues and eigenvectors of $A$, and $\varphi_{i}$ are the corresponding mode shapes. The eigenstructure $(\lambda, \varphi)$ is a canonical parameterization of system (1) and considered as the system parameter $\theta \in \mathbb{C}^{(r+1) n}$ with

$$
\theta \stackrel{\text { def }}{=}\left[\begin{array}{c}
\Lambda \\
\operatorname{vec}(\Phi)
\end{array}\right]
$$

where $\Lambda=\left[\begin{array}{lll}\lambda_{1} & \ldots & \lambda_{n}\end{array}\right]^{T}$ is the vector containing all eigenvalues, $\Phi=\left[\begin{array}{lll}\varphi_{1} & \ldots & \varphi_{n}\end{array}\right]$ is the matrix whose columns are the mode shapes and vec denotes the vectorization operator.

\subsection{Subspace-based residual vector}

In $[12,13]$ a residual function was proposed to detect changes in the eigenstructure $\theta$ from the measurements $y_{k}$ without actually identifying the eigenstructure in the possibly damaged state. The considered residual is associated with a covariance-driven output-only subspace identification algorithm. Let $G=\mathbf{E}\left(x_{k+1} y_{k}^{T}\right)$ be the cross-covariance between the states and the outputs, let $R_{i}=\mathbf{E}\left(y_{k} y_{k-i}^{T}\right)=C A^{i-1} G$ be the theoretic output covariances and

$$
\mathcal{H}_{p+1, q} \stackrel{\text { def }}{=}\left[\begin{array}{cccc}
R_{1} & R_{2} & \ldots & R_{q} \\
R_{2} & R_{3} & \ldots & R_{q+1} \\
\vdots & \vdots & \ddots & \vdots \\
R_{p+1} & R_{p+2} & \ldots & R_{p+q}
\end{array}\right] \stackrel{\text { def }}{=} \operatorname{Hank}\left(R_{i}\right)
$$

be the theoretic block Hankel matrix. It possesses the well-known factorization property

$$
\mathcal{H}_{p+1, q}=O_{p+1} C_{q}
$$

into the matrices of observability and controllability

$$
O_{p+1}=\left[\begin{array}{c}
C \\
C A \\
\vdots \\
C A^{p}
\end{array}\right], \quad C_{q}=\left[\begin{array}{llll}
G & A G & \ldots & A^{q-1} G
\end{array}\right] .
$$


From the observability matrix $O_{p+1}$, the matrices $C$ and $A$ could be recovered [16] and subsequently the system parameter $\theta$. However, a residual function based on $O_{p+1}$ (and $\mathcal{H}_{p+1, q}$ using factorization property (3)) is used in the following, instead of doing the system identification step.

Denote the system parameter in a reference state as $\theta_{0}$ (nominal model) and in the current state of the system as $\theta$, as defined in (2). The residual function for a damage detection test from $[12,13]$ compares the system parameter $\theta_{0}$ to data measured from the system $\theta$, which requires the identification of $\theta_{0}$ once from data of the reference state of the structure, while $\theta$ is not identified from the currently tested state. From $\theta_{0}$, the observability matrix $O_{p+1}\left(\theta_{0}\right)$ is obtained in the modal basis and its left null space $S\left(\theta_{0}\right)$ is computed, e.g. by a singular value decomposition of $O_{p+1}\left(\theta_{0}\right)$, such that

$$
S\left(\theta_{0}\right)^{T} O_{p+1}\left(\theta_{0}\right)=0 .
$$

Then, $S\left(\theta_{0}\right)$ is also the left null space of the block Hankel matrix $\mathcal{H}_{p+1, q}$ in the reference state because of factorization property (3), and the characteristic property of a system in the reference state corresponding to $\theta=\theta_{0}$ writes

$$
S\left(\theta_{0}\right)^{T} \mathcal{H}_{p+1, q}=0
$$

Using measured data $\left(y_{k}\right)_{k=1, \ldots, N}$, a consistent estimate $\widehat{\mathcal{H}}_{p+1, q}$ is obtained from the empirical output covariances

$$
\widehat{R}_{i}=\frac{1}{N} \sum_{k=1}^{N} y_{k} y_{k-i}^{T}, \quad \widehat{\mathcal{H}}_{p+1, q}=\operatorname{Hank}\left(\widehat{R}_{i}\right) .
$$

To decide whether the measured data corresponds to $\theta_{0}$ or not, the residual vector $\zeta_{N}^{Q}$ with

$$
\zeta_{N}^{Q}=\sqrt{N} \operatorname{vec}\left(S\left(\theta_{0}\right)^{T} \widehat{\mathcal{H}}_{p+1, q}\right)
$$

is defined $[12,13]$. The index $Q$ of the residual vector $\zeta_{N}^{Q}$ indicates the excitation covariance of the system, for which the measured data $\left(y_{k}\right)_{k=1, \ldots, N}$ are used for the computation of $\widehat{\mathcal{H}}_{p+1, q}$ in (5). It is tested if this residual function is significantly different from zero or not, corresponding to a test between the hypotheses

$$
\begin{array}{ll}
\mathbf{H}_{0}: \theta=\theta_{0} & \text { (reference system), } \\
\mathbf{H}_{1}: \theta \neq \theta_{0} & \text { (faulty system). }
\end{array}
$$

For mathematical convenience the alternative hypothesis $\mathbf{H}_{1}$ can be formulated as $\theta=\theta_{0}+\delta \theta / \sqrt{N}$ based on the asymptotic local approach for change detection [17], where $\delta \theta$ is an unknown but fixed change vector. In this formulation emphasis is put on the detection of small changes, and indeed very small changes in the system parameter $\theta$ can be detected with this statistical framework if $N$ is large enough. Also, this framework allows to show asymptotic normality for $N \rightarrow \infty$ of the residual function [12] with

$$
\zeta_{N}^{Q} \longrightarrow \begin{cases}\mathcal{N}\left(0, \Sigma_{\zeta}^{Q}\right) & \text { under } \mathbf{H}_{0}, \\ \mathcal{N}\left(\mathcal{J}_{\zeta}^{Q} \delta \theta, \Sigma_{\zeta}^{Q}\right) & \text { under } \mathbf{H}_{1}\end{cases}
$$

where $\mathcal{J}_{\zeta}^{Q}$ and $\Sigma_{\zeta}^{Q}$ are the asymptotic sensitivity and covariance of the residual $\zeta_{N}^{Q}$. The convergence in (8) means that the residual vector can be approximated by a Gaussian random vector when the number of samples $N$ is large, thus being completely defined by its mean and covariance. The mean of this asymptotic Gaussian vector is zero in the reference state and different from zero in the damaged state, and its covariance is the same under both hypotheses. A decision between the hypotheses $\mathbf{H}_{0}$ and $\mathbf{H}_{1}$ is achieved through a generalized likelihood ratio (GLR) test [12, 17]. Before actually deriving this test, the influence of a change in the excitation covariance $Q$ on the properties of the residual vector is investigated.

\section{Hypothesis test under changing excitation properties}

\subsection{Impact of changing excitation properties}

In practice, the excitation covariance $Q$ may change between different measurement sessions of the system due to different environmental factors (wind, traffic, ...), while the excitation is still assumed to be stationary during one 
measurement. A change in the excitation covariance $Q$ leads to a change in the cross-covariance between states and outputs $G$ and thus in the Hankel matrix $\mathcal{H}_{p+1, q}$ due to factorization (3)-(4). Subsequently, its estimate $\widehat{\mathcal{H}}_{p+1, q}$ and thus the residual function $\zeta_{N}^{Q}$ in (6) are changed, even if the eigenstructure of the system remains unchanged. Thus, the asymptotic residual's sensitivity and covariance $\mathcal{J}_{\zeta}^{Q}$ and $\Sigma_{\zeta}^{Q}$ are indeed dependent on the excitation covariance $Q$ and change when $Q$ changes. Note however that the system parameters $\theta_{0}$ or $\theta$ are independent of $Q$. Thus, the parametric observability matrix $O\left(\theta_{0}\right)$ and its left null space $S\left(\theta_{0}\right)$ that are obtained in the reference state are also independent of $Q$.

Special care must be taken about changing excitation properties between the reference state and the currently tested state of the structure for the derivation of a hypothesis test. Denote $Q$ as the covariance of the excitation under which the data in the reference state of the structure was recorded, and denote $\widetilde{Q}$ as the excitation covariance in the current state that is tested for damage. They cannot be measured and are different in general. Thus, consistent estimates of $\mathcal{J}_{\zeta}^{Q}$ and $\Sigma_{\zeta}^{Q}$ of the asymptotic sensitivity and covariance of the residual $\zeta_{N}^{Q}$ obtained in the reference state might not correspond to estimates of $\mathcal{J}_{\zeta}^{\widetilde{Q}}$ and $\Sigma_{\zeta}^{\widetilde{Q}}$ related to $\zeta_{N}^{\widetilde{Q}}$ obtained from another measurement in the tested (possibly damaged) state.

\subsection{Hypothesis test}

Now, recall the GLR test for a decision between hypotheses $\mathbf{H}_{0}$ and $\mathbf{H}_{1}$ in (7): let $Z$ be a Gaussian vector and let $p_{\theta_{0}}$ and $p_{\theta}$ be its asymptotic probability density functions under $\mathbf{H}_{0}$ and $\mathbf{H}_{1}$, respectively. Then, the GLR test writes as

$$
\operatorname{GLR}(Z)=-2 \log \frac{p_{\theta_{0}}(Z)}{\sup _{\theta \in \mathbf{H}_{1}} p_{\theta}(Z)} .
$$

First we show that the asymptotic residual distributions of (8) with different excitation covariances $Q$ and $\widetilde{Q}$ between reference state (in $p_{\theta_{0}}$ ) and tested state (in $p_{\theta}$ ), respectively, are incompatible in this GLR test. In this case it holds

$$
\begin{aligned}
G L R(Z) & =Z^{T}\left(\Sigma_{\zeta}^{Q}\right)^{-1} Z+\sup _{\theta \in \mathbf{H}_{1}}\left(-\left(Z-\mathcal{J}_{\zeta}^{\widetilde{Q}} \delta \theta\right)^{T}\left(\Sigma_{\zeta}^{\widetilde{Q}}\right)^{-1}\left(Z-\mathcal{J}_{\zeta}^{\widetilde{Q}} \delta \theta\right)\right) \\
& =Z^{T}\left(\Sigma_{\zeta}^{Q}\right)^{-1} Z-Z^{T}\left(\Sigma_{\zeta}^{\widetilde{Q}}\right)^{-1} Z+Z^{T}\left(\Sigma_{\zeta}^{\widetilde{Q}}\right)^{-1} \mathcal{J}_{\zeta}^{\widetilde{Q}}\left(\left(\mathcal{J}_{\zeta}^{\widetilde{Q}}\right)^{T}\left(\Sigma_{\zeta}^{\widetilde{Q}}\right)^{-1} \mathcal{J}_{\zeta}^{\widetilde{Q}}\right)^{-1}\left(\mathcal{J}_{\zeta}^{\widetilde{Q}}\right)^{T}\left(\Sigma_{\zeta}^{\widetilde{Q}}\right)^{-1} Z .
\end{aligned}
$$

If $Q \neq \widetilde{Q}$ the first two terms do not cancel and the GLR expression does not boil down to a $\chi^{2}$-distributed variable, which would be easy to evaluate. Moreover, the expression demands the residual covariance in both the reference and tested state. Thus, a comparison of the residual vector to properties of the reference state under a different excitation covariance is inconvenient in this setting.

However, it is not necessary to use properties of the reference state under a different excitation covariance in the GLR test. The asymptotic residual distributions under $\mathbf{H}_{0}$ and $\mathbf{H}_{1}$ can both be compared in the tested state (corresponding to excitation covariance $\widetilde{Q}$ ) in the GLR test for a decision if the newly computed residual $\zeta_{N}^{\widetilde{Q}}$ follows distribution $\mathcal{N}\left(0, \Sigma_{\zeta}^{\widetilde{Q}}\right)$ or $\mathcal{N}\left(\mathcal{J}_{\zeta}^{\widetilde{Q}} \delta \theta, \Sigma_{\zeta}^{\widetilde{Q}}\right)$. The respective GLR yields

$$
\begin{aligned}
G L R(Z) & =Z^{T}\left(\Sigma_{\zeta}^{\widetilde{Q}}\right)^{-1} Z-Z^{T}\left(\Sigma_{\zeta}^{\widetilde{Q}}\right)^{-1} Z+Z^{T}\left(\Sigma_{\zeta}^{\widetilde{Q}}\right)^{-1} \mathcal{J}_{\zeta}^{\widetilde{Q}}\left(\left(\mathcal{J}_{\zeta}^{\widetilde{Q}}\right)^{T}\left(\Sigma_{\zeta}^{\widetilde{Q}}\right)^{-1} \mathcal{J}_{\zeta}^{\widetilde{Q}}\right)^{-1}\left(\mathcal{J}_{\zeta}^{\widetilde{Q}}\right)^{T}\left(\Sigma_{\zeta}^{\widetilde{Q}}\right)^{-1} Z \\
& =Z^{T}\left(\Sigma_{\zeta}^{\widetilde{Q}}\right)^{-1} \mathcal{J}_{\zeta}^{\widetilde{Q}}\left(\left(\mathcal{J}_{\zeta}^{\widetilde{Q}}\right)^{T}\left(\Sigma_{\zeta}^{\widetilde{Q}}\right)^{-1} \mathcal{J}_{\zeta}^{\widetilde{Q}}\right)^{-1}\left(\mathcal{J}_{\zeta}^{\widetilde{Q}}\right)^{T}\left(\Sigma_{\zeta}^{\widetilde{Q}}\right)^{-1} Z .
\end{aligned}
$$

This expression is $\chi^{2}$-distributed with $\operatorname{rank}\left(\mathcal{J}_{\zeta}^{\widetilde{Q}}\right)$ degrees of freedom and non-centrality parameter zero under $\mathbf{H}_{0}$ and $\delta \theta^{T} F \delta \theta$ under $\mathbf{H}_{1}$, where $F=\left(\mathcal{J}_{\zeta}^{\widetilde{Q}}\right)^{T}\left(\Sigma_{\zeta}^{\widetilde{Q}}\right)^{-1} \mathcal{J}_{\zeta}^{\widetilde{Q}}$ is the Fisher information on $\theta_{0}$ contained in $Z$. Thus, the evaluation of such a test expression is easy as it requires only a comparison to a threshold to decide between $\mathbf{H}_{0}$ and $\mathbf{H}_{1}$. Since in practice only estimates are available, we compute the asymptotically $\chi^{2}$-distributed expression

$$
\chi_{N}^{2}=\left(\zeta_{N}^{\widetilde{Q}}\right)^{T}\left(\widehat{\Sigma}_{\zeta}^{\widetilde{Q}}\right)^{-1} \widehat{\mathcal{J}}_{\zeta}^{\widetilde{Q}}\left(\left(\widehat{\mathcal{J}}_{\zeta}^{\widetilde{Q}}\right)^{T}\left(\widehat{\Sigma}_{\zeta}^{\widetilde{Q}}\right)^{-1} \widehat{\mathcal{J}}_{\zeta}^{\widetilde{Q}}\right)^{-1}\left(\widehat{\mathcal{J}}_{\zeta}^{\widetilde{Q}}\right)^{T}\left(\widehat{\Sigma}_{\zeta}^{\widetilde{Q}^{-1}}\right)^{-1} \zeta_{N}^{\widetilde{Q}}
$$


as our damage detection test, where $\widehat{\mathcal{J}}_{\zeta}^{\widetilde{Q}}$ and $\widehat{\Sigma}_{\zeta}^{\widetilde{Q}}$ are estimates of the sensitivity and covariance of residual $\zeta_{N}^{\widetilde{Q}}$ in the tested state. Their computation is described in Appendix A.

Thus, the damage detection test (9) requires the computation of $\widehat{\mathcal{J}}_{\zeta}^{\widetilde{Q}}$ and $\widehat{\Sigma_{\zeta}} \widetilde{Q}$ in the currently tested state when the excitation covariance is changing, excluding the estimates in the reference state under the excitation covariance $Q$. In the expression (9), the only mention of the reference state $\theta_{0}$ is the left null space $S\left(\theta_{0}\right)$ that is independent of the excitation covariance $Q$ or $\widetilde{Q}$ in the residual function (6). Then, the residual, its sensitivity and its covariance are computed on the same data set and are coherent.

\subsection{Special case: constant excitation covariance}

If the excitation covariance remains the same between the reference and tested states, i.e. $Q=\widetilde{Q}$, it holds $\mathcal{J}_{\zeta}^{Q}=\mathcal{J}_{\zeta}^{\widetilde{Q}}$ and $\Sigma_{\zeta}^{Q}=\Sigma_{\zeta}^{\widetilde{Q}}$. Only in this case it is sufficient to estimate the residual's sensitivity and covariance from a measurement in the reference state, and the index $Q$ or $\widetilde{Q}$ can be dropped from all variables. The damage detection test can then be performed by computing

$$
\chi_{N}^{2}=\zeta_{N}^{T} \widehat{\Sigma}_{\zeta}^{-1} \widehat{\mathcal{J}}_{\zeta}\left(\widehat{\mathcal{J}}_{\zeta}^{T} \widehat{\Sigma}_{\zeta}^{-1} \widehat{\mathcal{J}}_{\zeta}\right)^{-1} \widehat{\mathcal{J}}_{\zeta}^{T} \widehat{\Sigma}_{\zeta}^{-1} \zeta_{N}
$$

which should be compared to a threshold to decide between both hypotheses $\mathbf{H}_{0}$ and $\mathbf{H}_{1}$. This test was derived in $[12,13]$, where estimates $\widehat{\mathcal{J}}_{\zeta}$ and $\widehat{\Sigma}_{\zeta}$ are only obtained once in the reference state, as the excitation covariance $Q$ was implicitly assumed constant between different structural states.

However, this strategy cannot be used in the case of changing excitation covariance as shown in the previous section. Instead, the computation of the residual's sensitivity and covariance using new data from the unknown currently tested state in the GLR test in (9) indeed presents a solution to the problem of changing excitation between measurements. However, the computation of the residual's covariance is more convenient using data from the reference state of the structure, where usually long data sets are available in practical applications. Then, better covariance estimates can be obtained from data in the reference state than on possibly shorter data sets in the currently tested state. Moreover, the computation of the covariance and its inverse is computationally taxing. Hence, it would be favorable to compute it only once from data of the reference state, instead of recomputing it in every tested structural state. This is only possible for a residual, whose covariance does not change when $Q$ changes between two measurement sessions.

\section{Residual and test robust to change in excitation covariance}

A new possibility to compensate a change in the excitation covariance $Q$ between the reference and the possibly damaged states is the use of a residual function that is robust to these changes. In this section, a hypothesis test on such a residual is derived.

The subspace-based residual vector in Section 2 is based on the property that the system parameter $\theta_{0}$ agrees with a Hankel matrix $\mathcal{H}_{p+1, q}$ if and only if $\mathcal{O}_{p+1}\left(\theta_{0}\right)$ and $\mathcal{H}_{p+1, q}$ have the same left null space $S\left(\theta_{0}\right)$. The same property holds for the left singular vectors of $\mathcal{H}_{p+1, q}$ : let $U_{1}$ be the matrix of the left singular vectors obtained from an SVD of $\mathcal{H}_{p+1, q}$. Then, $\mathcal{H}_{p+1, q}, O_{p+1}\left(\theta_{0}\right)$ and $U_{1}$ define the same subspace. Thus, $O_{p+1}\left(\theta_{0}\right)$ and $U_{1}$ have the same left null space $S\left(\theta_{0}\right)$, and the characteristic property of the system in the reference state writes as

$$
S\left(\theta_{0}\right)^{T} U_{1}=0
$$

As $U_{1}$ is a matrix with orthonormal columns, it can be regarded as independent of the excitation $Q$, which qualifies its use for a residual function that is robust to changes in the excitation covariance. Matrix $U_{1}$ is defined by a unique SVD to ensure no changing modal basis, e.g. by forcing the first entry in each singular vector to be positive.

The robust residual function is then defined as follows. Let $\widehat{\mathcal{H}}_{p+1, q}$ be an estimate of the Hankel matrix from a data sample $\left(y_{k}\right)_{k=1, \ldots, N}$ corresponding to the current, possibly damaged state $\theta$ under the unknown excitation covariance $\widetilde{Q}$. From an SVD

$$
\widehat{\mathcal{H}}_{p+1, q}=\left[\begin{array}{ll}
\widehat{U}_{1} & \widehat{U}_{0}
\end{array}\right]\left[\begin{array}{cc}
\widehat{\Delta}_{1} & 0 \\
0 & \widehat{\Delta}_{0}
\end{array}\right]\left[\begin{array}{c}
\widehat{V}_{1}^{T} \\
\widehat{V}_{0}^{T}
\end{array}\right]
$$


the matrix $\widehat{U}_{1}$ is obtained, whose number of columns is the system order $n$. Note that the singular values in $\widehat{\Delta}_{0}$ are very small and tend to zero for $N \rightarrow \infty$. Then, a residual vector that is robust to a change in the excitation covariance is defined as

$$
\xi_{N} \stackrel{\text { def }}{=} \sqrt{N} \operatorname{vec}\left(S\left(\theta_{0}\right)^{T} \widehat{U}_{1}\right),
$$

similarly as in [15]. In the following, the framework of the asymptotic local approach from the conventional damage detection test [12] described in Section 2.2 is applied for the precise computation of the residual's covariance and sensitivity for a correct statistical evaluation. We now prove that the robust residual $\xi_{N}$ is asymptotically Gaussian for $N \rightarrow \infty$. Let the SVDs of the Hankel matrix $\mathcal{H}_{p+1, q}=U_{1} \Delta_{1} V_{1}^{T}$ and its estimate $\widehat{\mathcal{H}}_{p+1, q}$ in (11) be given. From [18] and [19, Corollary 1] follows that $\widehat{U}_{1}$ is asymptotically Gaussian with

$$
\sqrt{N} \operatorname{vec}\left(\widehat{U}_{1}-U_{1}\right) \rightarrow \mathcal{N}\left(0, \Sigma_{U_{1}}\right)
$$

where $\Sigma_{U_{1}}=\lim _{N \rightarrow \infty} \operatorname{cov}\left(\sqrt{N} \operatorname{vec}\left(\widehat{U}_{1}\right)\right)$. It follows that the residual $\xi_{N}$ is asymptotically Gaussian, with asymptotic covariance $\Sigma_{\xi}=\left(I \otimes S^{T}\right) \Sigma_{U_{1}}(I \otimes S)$. Analogous to the proof of asymptotic normality of residual $\zeta_{N}[12,17]$ it follows

$$
\xi_{N} \longrightarrow \begin{cases}\mathcal{N}\left(0, \Sigma_{\xi}\right) & \text { under } \mathbf{H}_{0} \\ \mathcal{N}\left(\mathcal{J}_{\xi} \delta \theta, \Sigma_{\xi}\right) & \text { under } \mathbf{H}_{1}\end{cases}
$$

where $\mathcal{J}_{\xi}$ and $\Sigma_{\xi}$ are the asymptotic sensitivity and covariance of the residual $\xi_{N}$ and the hypotheses $\mathbf{H}_{0}$ and $\mathbf{H}_{1}$ are defined in (7). The GLR test to decide between the hypotheses $\mathbf{H}_{0}$ and $\mathbf{H}_{1}$ can thus be achieved through the asymptotic $\chi^{2}$-test statistics

$$
\gamma_{N}^{2}=\xi_{N}^{T} \widehat{\Sigma}_{\xi}^{-1} \widehat{\mathcal{J}}_{\xi}\left(\widehat{\mathcal{J}}_{\xi}^{T} \widehat{\Sigma}_{\xi}^{-1} \widehat{\mathcal{J}}_{\xi}\right)^{-1} \widehat{\mathcal{J}}_{\xi}^{T} \widehat{\Sigma}_{\xi}^{-1} \xi_{N}
$$

which should be compared to a threshold, and where $\widehat{\mathcal{J}}_{\xi}$ and $\widehat{\Sigma}_{\xi}$ are consistent estimates of $\mathcal{J}_{\xi}$ and $\Sigma_{\xi}$. Due to the construction of the residual function, the asymptotic sensitivity $\mathcal{J}_{\xi}$ and covariance $\Sigma_{\xi}$ of residual $\xi_{N}$ do not depend on the excitation covariance $Q$. Their estimates can thus be computed once on data from the reference state and be used for the damage detection test under a possibly different excitation $\widetilde{Q}$. The computation of the new residual sensitivity and covariance is detailed in Appendix B.

\section{Non-parametric damage detection tests}

In [14] a non-parametric version of the damage detection test is proposed, where the system parameter $\theta_{0}$ does not need to be known explicitly in the reference state. Instead of using the null space $S\left(\theta_{0}\right)$ on the parameterized observability matrix $O_{p+1}\left(\theta_{0}\right)$, an empirical (non-parametric) null space $\bar{S}$ is computed on an estimated block Hankel matrix $\widehat{\mathcal{H}}_{p+1, q}^{\text {(reference) }}$ from data in the reference state using e.g. an SVD. The characteristic property of a system in the reference state writes then as

$$
\bar{S}^{T} \widehat{\mathcal{H}}_{p+1, q}=0
$$

The resulting non-parametric residual function and damage detection test are then entirely based on the measured data and no parametric model is used. Like this, the non-parametric test may be more convenient than the parametric one, as no system identification is necessary in the reference state to retrieve $\theta_{0}$. On the other side, the non-parametric approach does not allow the choice of modes in the system parameter $\theta_{0}$ that are monitored for a change due to damage and the whole system response is taken into account.

In this section, the parametric damage detection tests from Sections 3.2, 3.3 and 4 are simplified to be used as non-parametric tests.

\subsection{Test with conventional residual under changing excitation covariance}

Based on the residual function presented in Section 2.2 in (6), the non-parametric residual function is consequently defined as

$$
\bar{\zeta}_{N}^{Q}=\sqrt{N} \operatorname{vec}\left(\bar{S}^{T} \widehat{\mathcal{H}}_{p+1, q}\right)
$$


where $\widehat{\mathcal{H}}_{p+1, q}$ is computed on data corresponding to excitation covariance $Q$. Let $\Sigma_{\zeta}^{Q}$ be the asymptotic covariance of $\bar{\zeta}_{N}^{Q}$. Analogously to Section 3.2, the residual $\bar{\zeta}_{N}^{Q}$ is asymptotically Gaussian for any $Q$. Let $\widetilde{Q}$ be the excitation covariance in the currently tested state. Then, a non-parametric test to decide if $\bar{\zeta}_{N}^{\widetilde{Q}}$ is significantly different from zero or not boils down to [14]

$$
\bar{\chi}_{N}^{2}=\left(\bar{\zeta}_{N}^{\widetilde{Q}}\right)^{T}\left(\widehat{\Sigma}_{\zeta}^{\widetilde{Q}}\right)^{-1} \bar{\zeta}_{N}^{\widetilde{Q}},
$$

which should be compared to a threshold, and where $\widehat{\Sigma}_{\zeta}^{\widetilde{Q}}$ is a consistent estimate of $\Sigma_{\zeta}^{\widetilde{Q}}$ computed on data in the currently tested state. Its computation follows the covariance computation of the parametric residual function and is described in Appendix A.1.

\subsection{Test with conventional residual under constant excitation covariance}

In Section 3.3, the conventional damage detection test was stated under a constant excitation covariance $Q=\widetilde{Q}$ in the currently tested state as in the reference state. In this case, the dependence of the residual and its covariance on $Q$ can be dropped, and the residual covariance is estimated only once in the reference state as in [14]. The non-parametric counterpart thus writes analogously to (16) as

$$
\bar{\chi}_{N}^{2}=\bar{\zeta}_{N}^{T} \widehat{\Sigma}_{\zeta}^{-1} \bar{\zeta}_{N}
$$

where $\bar{\zeta}_{N}$ is the residual function defined on the empirical null space $\bar{S}$ in (14), and $\widehat{\Sigma}_{\zeta}$ is the estimate of its covariance computed only once from data in the reference state as the excitation covariance is assumed to be constant for both the reference and the currently tested states.

\subsection{Test with new residual robust to changing excitation}

Analogous to Section 5.1, a non-parametric version of the damage detection test robust to excitation changes from Section 4 can be defined by using an empirical left null space $\bar{S}$ with $\bar{S}^{T} \widehat{\mathcal{H}}_{p+1, q}=0$ in the reference state. The characteristic property in the reference state writes then as

$$
\bar{S}^{T} \widehat{U}_{1}=0
$$

where $\widehat{U}_{1}$ is obtained from SVD (11) of a Hankel matrix $\widehat{\mathcal{H}}_{p+1, q}$ estimated on a data sample $\left(y_{k}\right)_{k=1, \ldots, N}$. Then, the non-parametric residual function is defined as

$$
\bar{\xi}_{N} \stackrel{\text { def }}{=} \sqrt{N} \operatorname{vec}\left(\bar{S}^{T} \widehat{U}_{1}\right) .
$$

Let $\Sigma_{\xi}$ be the asymptotic covariance of $\bar{\xi}_{N}$. Analogous to the parametric residual $\xi_{N}$, the residual $\bar{\xi}_{N}$ is asymptotically Gaussian and the $\chi^{2}$-test statistics (13) boils down to

$$
\bar{\gamma}_{N}^{2}=\bar{\xi}_{N}^{T} \widehat{\Sigma}_{\xi}^{-1} \bar{\xi}_{N},
$$

where $\widehat{\Sigma}_{\xi}$ is a consistent estimate of the asymptotic covariance of $\bar{\xi}_{N}$. Its computation is only necessary once in the reference state and is equivalent to the computation in Section Appendix B.1, where $S$ is replaced by $\bar{S}$.

\section{Numerical considerations}

In this section, special care is taken of numerical aspects of the computation of the $\chi^{2}$-test statistics that are used for the subspace-based damage detection tests. These issues are particularly important for a sensible and numerically robust implementation of the damage detection algorithms. Possible problems arise mainly due to the covariance matrix of the residual, which can be big and rank deficient, and hence its inversion in the tests is numerically critical. These computations appear in (9), (10) and (13), where they are of the form

$$
\chi^{2}=\Upsilon^{T} \widehat{\Sigma}^{-1} \widehat{\mathcal{J}}\left(\widehat{\mathcal{J}}^{T} \widehat{\Sigma}^{-1} \widehat{\mathcal{J}}\right)^{-1} \widehat{\mathcal{J}}^{T} \widehat{\Sigma}^{-1} \Upsilon
$$


or, in the non-parametric version in (15), (16) and (18), where they are of the form

$$
\chi^{2}=\Upsilon^{T} \widehat{\Sigma}^{-1} \Upsilon
$$

where $\Upsilon$ is the respective residual vector. Here only the numerical properties of their computation are considered and all subscripts and superscripts of the involved variables are skipped. Mainly three critical issues arise:

- The covariance matrix $\widehat{\Sigma}$ is not invertible. This happens when its dimensions are large, while the number of available samples is not sufficient to ensure full rank. In this case, all matrix inversion operations in (19) and (20) can be replaced by the pseudoinverse (denoted by ${ }^{\dagger}$ ) [20].

- The covariance matrix $\widehat{\Sigma}$ is extremely large and the computation of its inverse $\widehat{\Sigma}^{-1}$ or pseudoinverse $\widehat{\Sigma}^{\dagger}$ is unstable. The solution to this problem lies in the efficient computation of the square root (pseudo-)inverse $\widehat{\Sigma}^{-1 / 2}$ of the covariance matrix $\widehat{\Sigma}$, such that the relations

$$
\widehat{\Sigma}^{-1}=\left(\widehat{\Sigma}^{-1 / 2}\right)^{T} \widehat{\Sigma}^{-1 / 2}, \quad \text { or } \quad \widehat{\Sigma}^{\dagger}=\left(\widehat{\Sigma}^{-1 / 2}\right)^{T} \widehat{\Sigma}^{-1 / 2}
$$

hold in the full rank and rank deficient cases, respectively, while $\widehat{\Sigma}^{-1}$ or $\widehat{\Sigma}^{\dagger}$ themselves are not computed. In Appendix C. 1 it is shown how $\widehat{\Sigma}^{-1 / 2}$ can be efficiently computed directly from the data. Moreover, the large covariance matrix $\widehat{\Sigma}$ is not needed at all in this computation and the dimensions of $\widehat{\Sigma}^{-1 / 2}$ are much smaller than those of $\widehat{\Sigma}$ in the rank deficient case.

- The direct computation of $\chi^{2}$-tests (19) and (20) is numerically unstable. This is due to the bad conditioning of the covariance matrix. Matrix $\widehat{\Sigma}^{-1 / 2}$ in (21) plays a key role in the stable computation of the test value: a numerically stable computation of the non-parametric $\chi^{2}$-test (20) can be performed from

$$
\chi^{2}=\alpha^{T} \alpha \text { with } \alpha=\widehat{\Sigma}^{-1 / 2} \Upsilon .
$$

A numerically stable computation of the parametric test (19) was proposed in [21] and starts with the thin QR decomposition of the product

$$
\widehat{\Sigma}^{-1 / 2} \widehat{\mathcal{J}}=Q \mathcal{R}
$$

before computing the test from

$$
\chi^{2}=\alpha^{T} \alpha \text { with } \alpha=Q^{T} \widehat{\Sigma}^{-1 / 2} \Upsilon .
$$

Proofs and further details are given in Appendix C.2.

\section{Summary of the damage detection test robust to changes in the excitation covariance}

In this section, the computation details of the new robust damage detection test are summarized for the parametric test (Section 4) and the simplified non-parametric test (Section 5.3) taking into account the numerical considerations for the computation of Section 6. In a preprocessing step, the variables for the test are computed in the reference state. The available data in the reference state is always divided in two parts: one part of some length $N$ for the computation of the null space and the covariance of the test, and another part as a validation data set for the determination of a threshold. Then, the damage detection test can be performed sequentially on new data sets from a possibly damaged state.

\subsection{Parametric test}

\subsubsection{Preprocessing in the reference state}

1. Compute $\widehat{\mathcal{H}}_{p+1, q}$ on $N$ data samples in (5) and the singular vectors $\widehat{U}_{1}$ from the SVD of $\widehat{\mathcal{H}}_{p+1, q}$ in (11)

2. Do a system identification and select modes for the system parameter $\theta_{0}$ in (2)

3. Compute parametric observability matrix $O\left(\theta_{0}\right)$ in (A.4) and obtain null space $S\left(\theta_{0}\right)$ from SVD of $O\left(\theta_{0}\right)$

4. Compute sensitivity matrix $\widehat{\mathcal{J}}_{\xi}=\left(O_{p+1}\left(\theta_{0}\right)^{\dagger} \widehat{U}_{1} \otimes S\left(\theta_{0}\right)\right)^{T} O_{p+1}^{\prime}\left(\theta_{0}\right)$ in (B.2) with $O_{p+1}^{\prime}\left(\theta_{0}\right)$ from (A.5)-(A.6) 
5. Divide data of length $N$ into $n_{b}$ blocks of length $N_{b}$, compute $\widehat{\mathcal{H}}_{p+1, q}^{(j)}$ on each block and fill matrix $\mathcal{K}$ in (C.4) with the columns $h_{j}=\operatorname{vec}\left(\widehat{\mathcal{H}}_{p+1, q}^{(j)}-\widehat{\mathcal{H}}_{p+1, q}\right)$

6. Compute $\mathcal{J}_{\widehat{U}_{1}}$ in Proposition 1 (Appendix B.1), $\mathcal{A}=\left(I \otimes S\left(\theta_{0}\right)^{T}\right) \mathcal{J}_{\widehat{U}_{1}}$ and $\widehat{\Sigma}_{\xi}^{-1 / 2}=(\mathcal{A K})^{\dagger}$ in (C.5)

7. Perform the thin $\mathrm{QR}$ decomposition $\widehat{\Sigma}_{\xi}^{-1 / 2} \widehat{\mathcal{J}}_{\xi}=Q \mathcal{R}$ in (23) and compute the product $Q^{T} \widehat{\Sigma}_{\xi}^{-1 / 2}$

8. For each further reference data set,

- compute $\widehat{\mathcal{H}}_{p+1, q}$ on data set of some length $N$, the singular vectors $\widehat{U}_{1}$ from the SVD of $\widehat{\mathcal{H}}_{p+1, q}$ in (11) and residual $\xi_{N}=\sqrt{N} \operatorname{vec}\left(S\left(\theta_{0}\right)^{T} \widehat{U}_{1}\right)$ in (12),

- compute $\alpha=Q^{T} \widehat{\Sigma}_{\xi}^{-1 / 2} \xi_{N}$ and $\chi^{2}$-test value $\gamma_{N}^{2}=\alpha^{T} \alpha$ (Equations (13) and (24)),

and determine a threshold $t$ from the values $\gamma_{N}^{2}$ for a desired type I error.

The output of these preprocessing steps are the matrices $S\left(\theta_{0}\right)$, the product $Q^{T} \widehat{\Sigma}_{\xi}^{-1 / 2}$ and the threshold $t$ for the damage detection test.

\subsubsection{Testing a new data set for damage}

1. Compute $\widehat{\mathcal{H}}_{p+1, q}$ on data set of some length $N$, the singular vectors $\widehat{U}_{1}$ from the SVD of $\widehat{\mathcal{H}}_{p+1, q}$ in (11) and residual $\xi_{N}=\sqrt{N} \operatorname{vec}\left(S\left(\theta_{0}\right)^{T} \widehat{U}_{1}\right)$ in (12)

2. Compute $\alpha=Q^{T} \widehat{\Sigma}_{\xi}^{-1 / 2} \xi_{N}$ and $\chi^{2}$-test value $\gamma_{N}^{2}=\alpha^{T} \alpha$ (Equations (13) and (24))

3. If $\gamma_{N}^{2} \leq t$ : data set is classified as healthy; if $\gamma_{N}^{2}>t$ : data set is classified as damaged.

\subsection{Non-parametric test}

\subsubsection{Preprocessing in the reference state}

1. Compute $\widehat{\mathcal{H}}_{p+1, q}$ on $N$ data samples in (5)

2. Obtain null space $\bar{S}$ from SVD of $\widehat{\mathcal{H}}_{p+1, q}$ (e.g. as $\bar{S}=\widehat{U}_{0}$ in (11))

3. Divide data of length $N$ into $n_{b}$ blocks of length $N_{b}$, compute $\widehat{\mathcal{H}}_{p+1, q}^{(j)}$ on each block and fill matrix $\mathcal{K}$ in (C.4) with the columns $h_{j}=\operatorname{vec}\left(\widehat{\mathcal{H}}_{p+1, q}^{(j)}-\widehat{\mathcal{H}}_{p+1, q}\right)$

4. Compute $\mathcal{J}_{\widehat{U}_{1}}$ in Proposition 1 (Appendix B.1), $\mathcal{A}=\left(I \otimes \bar{S}^{T}\right) \mathcal{J}_{\widehat{U}_{1}}$ and $\widehat{\Sigma}_{\xi}^{-1 / 2}=(\mathcal{A K})^{\dagger}$ in (C.5)

5. For each further reference data set,

- compute $\widehat{\mathcal{H}}_{p+1, q}$ on data set of some length $N$, the singular vectors $\widehat{U}_{1}$ from the SVD of $\widehat{\mathcal{H}}_{p+1, q}$ in $(11)$ and residual $\bar{\xi}_{N}=\sqrt{N} \operatorname{vec}\left(\bar{S}^{T} \widehat{U}_{1}\right)$ in (17),

- compute $\alpha=\widehat{\Sigma}_{\xi}^{-1 / 2} \bar{\xi}_{N}$ and $\chi^{2}$-test value $\bar{\gamma}_{N}^{2}=\alpha^{T} \alpha$ (Equations (18) and (22)),

and determine a threshold $t$ from the values $\bar{\gamma}_{N}^{2}$ for a desired type I error.

The output of these preprocessing steps are the matrices $\bar{S}, \widehat{\Sigma}_{\xi}^{-1 / 2}$ and the threshold $t$ for the damage detection test.

\subsubsection{Testing a new data set for damage}

1. Compute $\widehat{\mathcal{H}}_{p+1, q}$ on data set of some length $N$, the singular vectors $\widehat{U}_{1}$ from the SVD of $\widehat{\mathcal{H}}_{p+1, q}$ in (11) and residual $\bar{\xi}_{N}=\sqrt{N} \operatorname{vec}\left(\bar{S}^{T} \widehat{U}_{1}\right)$ in (17)

2. Compute $\alpha=\widehat{\Sigma}_{\xi}^{-1 / 2} \bar{\xi}_{N}$ and $\chi^{2}$-test value $\bar{\gamma}_{N}^{2}=\alpha^{T} \alpha$ (Equations (18) and (22))

3. If $\bar{\gamma}_{N}^{2} \leq t$ : data set is classified as healthy; if $\bar{\gamma}_{N}^{2}>t$ : data set is classified as damaged. 


\section{Application}

The damage detection test derived in this paper that is robust to changes in the ambient excitation covariance is applied to three numerical examples and compared to the conventional tests to show their performance under different ambient excitation properties of a system. The $\chi^{2}$-test statistics of the damage detection algorithms are used in their non-parametric form from Section 5 for simplicity. Three variants were tested:

(a) Conventional test $\bar{\chi}_{N}^{2}=\bar{\zeta}_{N}^{T} \widehat{\Sigma}_{\zeta}^{-1} \bar{\zeta}_{N}$ in (16) based on [12,13], where the residual's covariance $\widehat{\Sigma}_{\zeta}$ is computed only once in the reference state (cf. also Section 3.3),

(b) Conventional test $\bar{\chi}_{N}^{2}=\left(\bar{\zeta}_{N}^{\widetilde{Q}}\right)^{T}\left(\widehat{\Sigma}_{\zeta}^{\widetilde{Q}}\right)^{-1} \bar{\zeta}_{N}^{\widetilde{Q}}$ in (15), where the residual's covariance $\widehat{\Sigma}_{\zeta}^{\widetilde{Q}}$ is computed each time in the tested state under the current excitation covariance $\widetilde{Q}$ (cf. also Section 3.2),

(c) New test $\bar{\gamma}_{N}^{2}=\bar{\xi}_{N}^{T} \widehat{\Sigma}_{\xi}^{-1} \bar{\xi}_{N}$ in (18), where the residual $\bar{\xi}_{N}$ is robust to changes in the ambient excitation covariance and the residual's covariance $\widehat{\Sigma}_{\xi}$ is computed only once in the reference state (cf. also Section 4). The corresponding algorithm is summarized in Section 7.2.

\subsection{Mass-spring chain}

First, a simulation study was made using a mass-spring model of six degrees of freedom (DOF), see Figure 1, which is observed at all six DOFs. Four cases of Gaussian stationary white noise excitation having a different covariance $\widetilde{Q}$ of the system were simulated:

1. $\widetilde{Q}=I_{6}$, represented by $\bigcirc$ in Figure 2 ,

2. $\widetilde{Q}=4^{2} I_{6}(\square)$,

3. $\widetilde{Q}=0.25^{2} I_{6}(\times)$,

4. $\widetilde{Q}=\operatorname{diag}(1,2,3,4,5,6)^{2}(+)$.

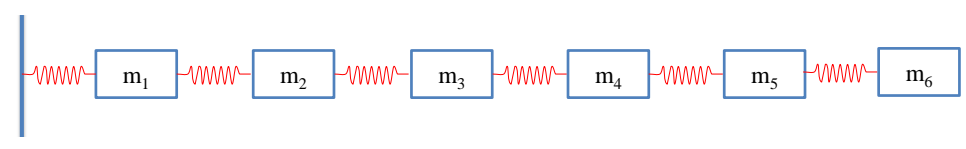

Figure 1: Simulated mass-spring chain.

Using this model, output-only data with $N=25000$ samples was generated to obtain measurements in the reference state with each of the different excitations. Then, the stiffness of spring 2 was reduced by $5 \%$ and a second time by $10 \%$ compared to the reference state, and the simulations were repeated with newly generated excitations.

For each state and excitation covariance, the experiment was repeated 4 times. The resulting $\chi^{2}$-test values of the three different tests (a)-(c) on this data are plotted in Figures 2(a)-2(c) in the following order: the first 16 test values are computed in the reference state, the next 16 values with 5\% stiffness reduction and the last 16 values with $10 \%$ stiffness reduction. In each of these states, 4 values correspond to one of the 4 different excitation covariances mentioned above. An empirical threshold (horizontal dashed line) to distinguish between reference states and damaged states is computed on the mean and variance of the $\chi^{2}$-test values of the 16 reference states.

As can be seen in Figure 2(a), the conventional $\chi^{2}$-test is strongly influenced by a different excitation covariance and no separation of the $\chi^{2}$-test values between reference and damaged states is possible. For each applied excitation covariance, the $\chi^{2}$-values increase with the damage, but if the excitation is unknown one cannot distinguish between the reference and damaged states. Recomputing the residual covariance on data of the currently tested state in Figure 2(b) already leads to a better separation between reference and damaged states, where less than $1 / 5$ of the values in the damaged states are below the threshold established by the reference states. With the new robust $\chi^{2}$-test in Figure $2(\mathrm{c})$, a clear separation between reference and damaged states is possible. Note also that the magnitude of damage is apparently linked to the obtained $\chi^{2}$-test values: Increasing the damage by factor 2 leads to $\chi^{2}$-test values that are approximately increased to factor 4 , which is to be expected. 


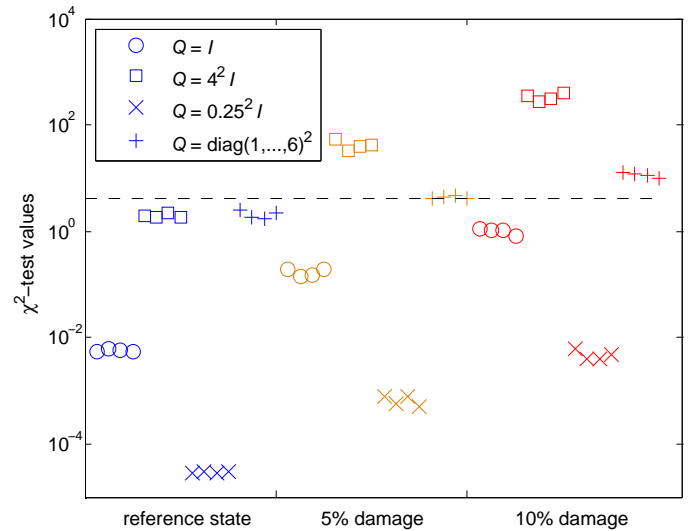

(a) Conventional test (16) with the residual's covariance computed once in the reference state (Section 3.3).

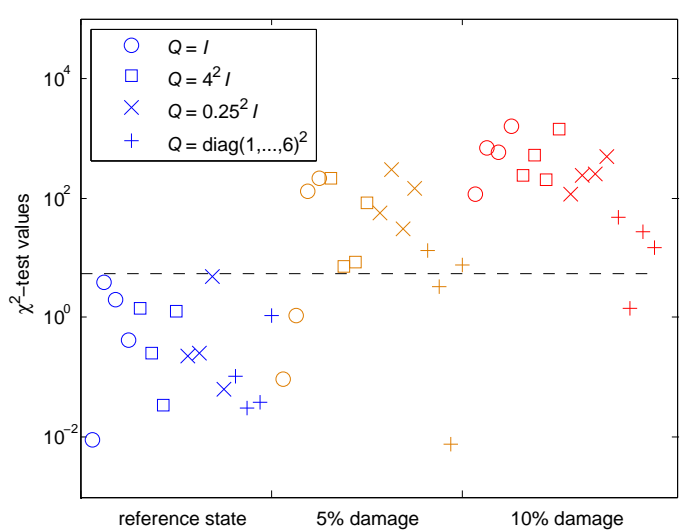

(b) Conventional test (15) with the residual's covariance computed in the tested state (Section 3.2).

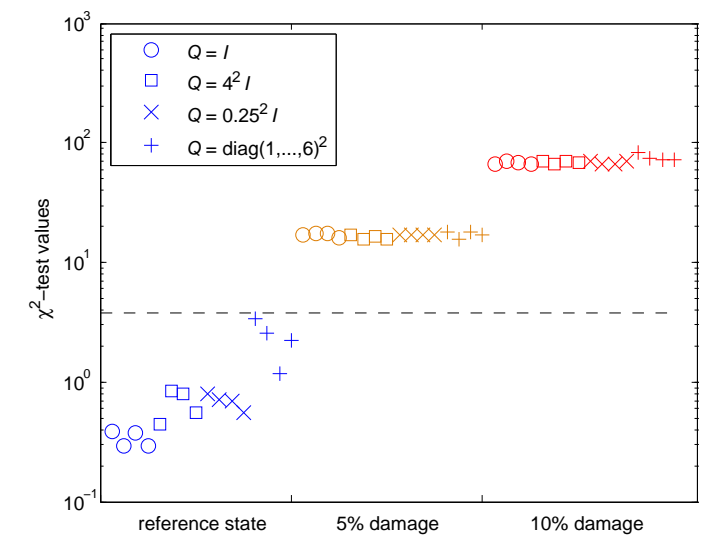

(c) New test (18) with the residual's covariance computed once in the reference state (Section 4).

Figure 2: Comparison of damage detection tests on mass-spring chain (log-scale).

\subsection{Truss structure}

For the simulation of a truss structure, more realistic conditions were considered. The truss model has 25 DOF (see Figure 3) and output data was generated at six sensor positions in vertical direction at the lower chord by exciting the structure at the same positions with white noise. The excitation noise at these six positions was generated with a diagonal covariance matrix $Q$, whose diagonal entries were randomly chosen from a uniform distribution in the interval $[1,36] .5 \%$ white noise were added on the generated outputs.

Damage was simulated by decreasing the stiffness of element 16 in a first step by $10 \%$ and in a second step by $20 \%$, leading to a decrease of the structure's natural frequencies of up to $1.0 \%$ and $2.2 \%$ compared to the reference state, respectively. In the reference state, the null space $\bar{S}$ and the residual covariance $\widehat{\Sigma}_{\zeta}$ (for test (a)) and $\widehat{\Sigma}_{\xi}$ (for test (c)) were computed on 10 data sets to set up the parameters of the damage detection tests.

\subsubsection{Robustness to changing excitation covariance}

In each structural state, data sets of length $N=25000$ at a sampling frequency of $50 \mathrm{~Hz}$ were generated. To compare the damage detection tests (a)-(c) under changing excitation, 100 new data samples were generated with 


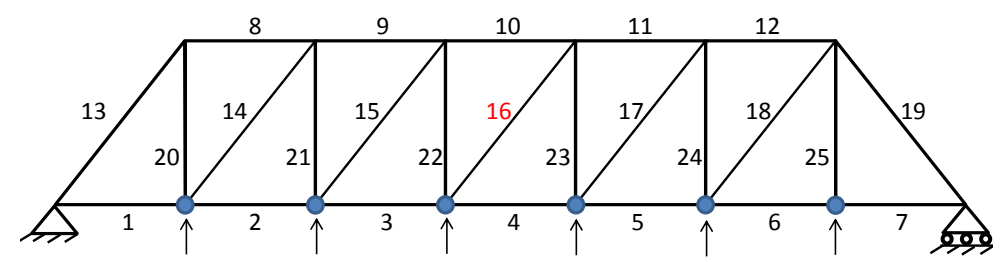

Figure 3: Truss structure with six sensors.

random excitation covariance in the reference and both damaged states, on which the three $\chi^{2}$-tests (a)-(c) were computed in Figure 4. For each of the compared tests, an empirical threshold was computed from the $\chi^{2}$-values of the reference state allowing a $5 \%$ type I error (horizontal dashed line).

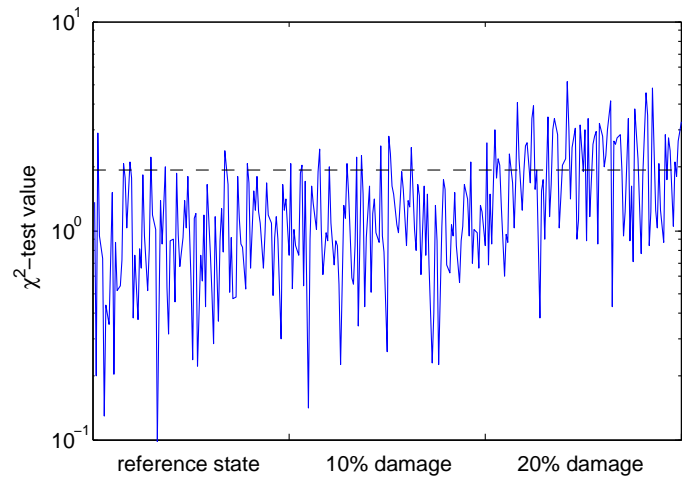

(a) Conventional test (16) with the residual's covariance computed once in the reference state (Section 3.3).

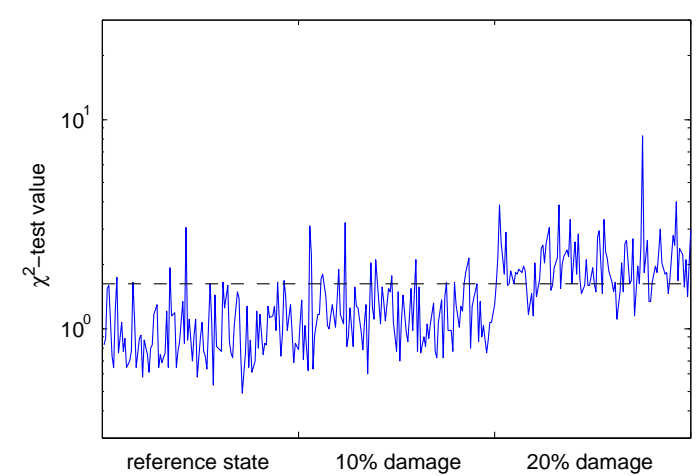

(b) Conventional test (15) with the residual's covariance computed in the tested state (Section 3.2).

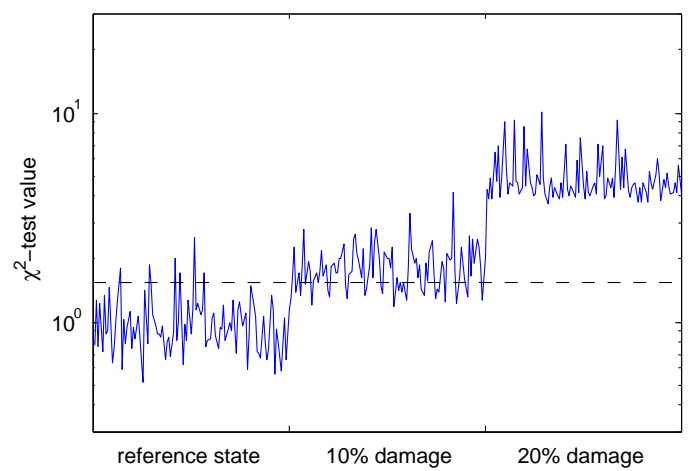

(c) New test (18) with the residual's covariance computed once in the reference state (Section 4).

Figure 4: Comparison of damage detection tests on truss structure (log-scale).

As can be seen in Figures 4(a) and 4(b), no satisfying separation between the reference and damaged state is possible with the conventional tests. Recomputing the covariance of the residual as in (15) in Figure 4(b) yields already better results than applying the conventional residual of (16) in Figure 4(a), as expected. However, the computation of the residual covariance is the numerically and statistically most critical part of the damage detection test. Recomputing 
$\widehat{\Sigma}$ all the time as in (15) requires a very precise estimation and therefore a sufficiently long data sample length for each new record. Otherwise, numerical errors on $\widehat{\Sigma}$ on each new record will deteriorate the algorithm's efficiency. This is a major argument towards choosing a reactive algorithm but also requiring only one computation of $\widehat{\Sigma}$ on a reference data set. Still some improvement can be seen over the conventional test (16) that is not designed for a changing excitation. Finally, the new damage detection test in Figure 4(c) manages to separate the reference state from the damaged states very well. The power of the test, which denotes the percentage of correct classification of data sets in the damaged condition as damaged, reaches $100 \%$ in the second damaged state.

The empirical probability densities of the $\chi^{2}$-test values have been obtained from multiple runs of the damage detection tests in Figure 5. While the conventional tests (a) and (b) show strongly overlapping distributions, the new test (c) shows well separated distributions in the three structural states. Also, it is clearly visible that the damage detection tests show the behavior of the $\chi^{2}$-distribution.

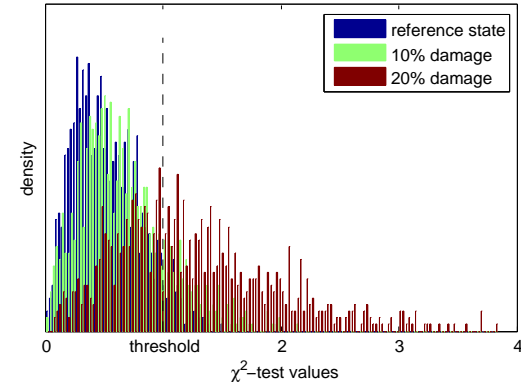

(a) Conventional test (16) with residual's covariance computed once in the reference state.

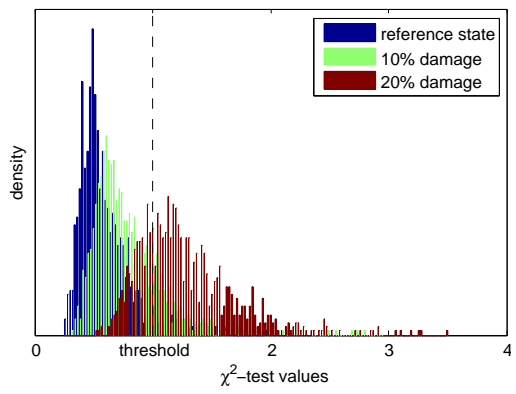

(b) Conventional test (15) with the residual's covariance computed in the tested state.

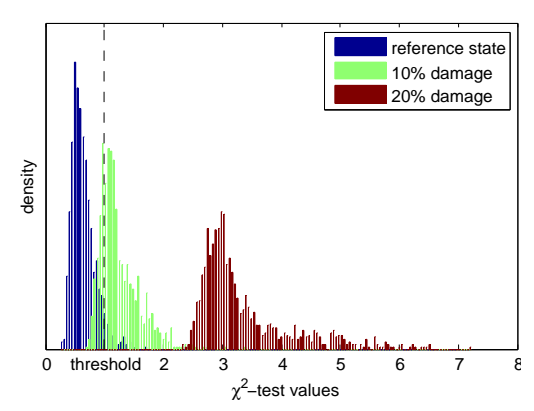

(c) New test (18) with the residual's covariance computed once in the reference state.

Figure 5: Histograms of $\chi^{2}$-test values from the different damage detection tests on truss structure using data samples of length $N=25000$.

After the preprocessing step in the reference state, the computation time of a $\chi^{2}$ value for a tested data set of length $N=25000$ was around $0.06 \mathrm{~s}$ for both tests (a) and (c), and around $0.8 \mathrm{~s}$ for test (b), as the covariance of the respective residual needs to be evaluated additionally in test (b). Note that the speed of all algorithms is improved by the numerical considerations in Section 6, in particular the computation of the inverse of the covariance for test (b).

\subsubsection{Impact of data length $N$}

From the formulation of the hypotheses $\mathbf{H}_{0}$ and $\mathbf{H}_{1}$ in Section 2.2 it follows that smaller damages can be detected when the data length $N$ increases, i.e. the damage detection tests get more reactive for longer data sets. This is verified on the simulated truss for all three algorithms (a)-(c), using $N=100000$ and $N=200000$ samples on the same damage scenarios as in the previous section. The empirical probability densities of the respective $\chi^{2}$-test values are presented in Figures 6 and 7, while the corresponding densities using $N=25000$ samples were obtained in Figure 5.

Comparing Figures 5-7, it can be seen that the distributions for the reference and the damaged states of the conventional test in (a) are always overlapping and no complete separation between the structural states can be achieved, even if the data length is increased, as the test is not designed for a changing excitation covariance. Re-computing the residual covariance of the conventional test in (b) leads to a better separation between the different structural states and the damaged states yield $\chi^{2}$-test values that are clearly different from zero. When the data length is increased, the separation between the structural states improves, and test (b) gets closer to its theoretical efficiency predicted by the local approach assumption. The new test (c) already achieved a good separation between the structural states for $N=25000$, and its efficiency also improves with the data length. It yields the most concentrated distributions that are distinctive for the different structural states. It can be concluded that conventional test (a) is definitely flawed when the excitation covariance changes between measurements, whereas a very large number of samples is required for test (b) to be usable for damage detection. In all cases, the new test (c) successfully detects damage at different levels under excitation changes, even using a reasonably low number of samples. The power of the test for the different sample lengths is summarized in Table 1. 


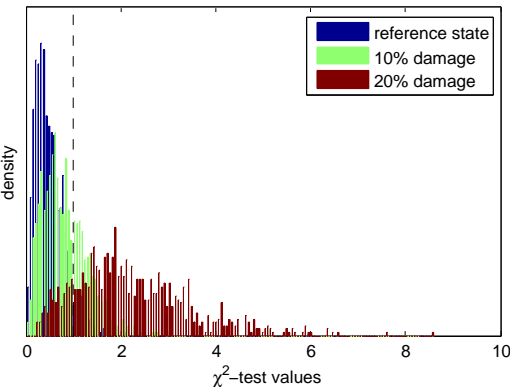

(a) Conventional test (16) with residual's covariance computed once in the reference state.

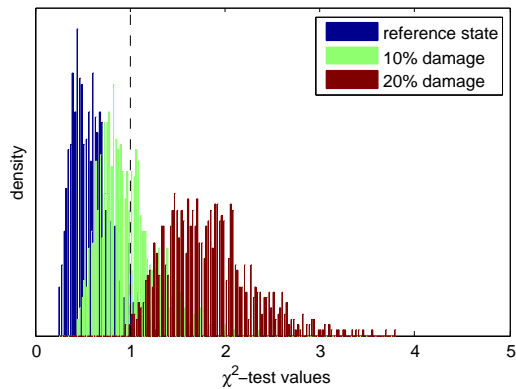

(b) Conventional test (15) with the residual's covariance computed in the tested state.

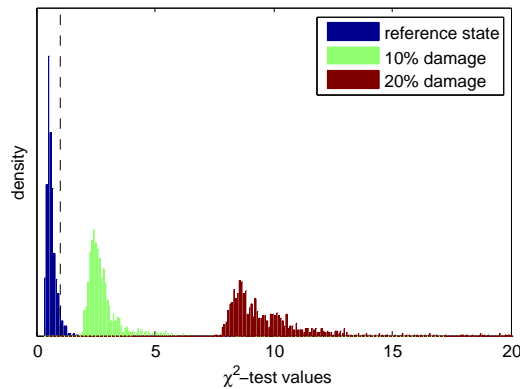

(c) New test (18) with the residual's covariance computed once in the reference state.

Figure 6: Histograms of $\chi^{2}$-test values from the different damage detection tests on truss structure using data samples of length $N=100000$.

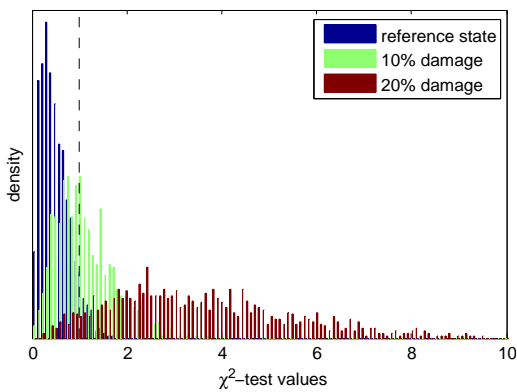

(a) Conventional test (16) with residual's covariance computed once in the reference state.

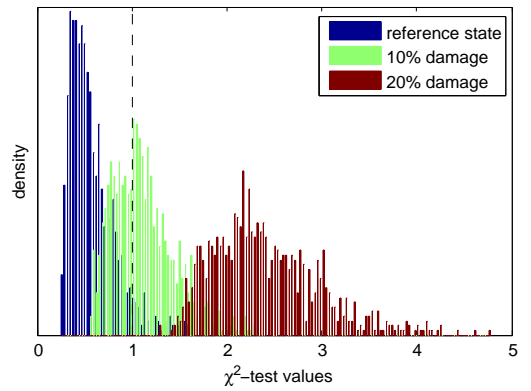

(b) Conventional test (15) with the residual's covariance computed in the tested state.

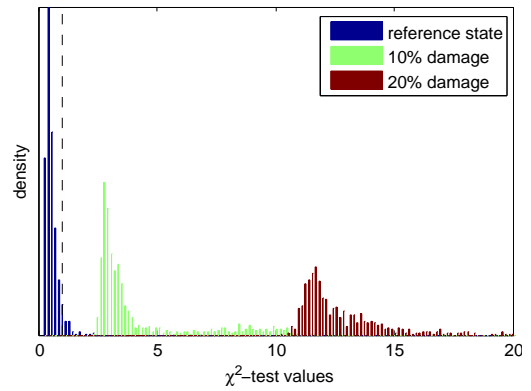

(c) New test (18) with the residual's covariance computed once in the reference state.

Figure 7: Histograms of $\chi^{2}$-test values from the different damage detection tests on truss structure using data samples of length $N=200000$.

Table 1: Power of the damage detection tests (in \%) for different data lengths and 5\% type I error.

\begin{tabular}{l|rrr|rrr} 
& \multicolumn{3}{|c|}{$10 \%$ damage on bar 16} & \multicolumn{4}{|c}{$20 \%$ damage on bar 16} \\
& (a) & (b) & (c) & (a) & (b) & (c) \\
\hline$N=25000$ & 14 & 13 & 65 & 55 & 62 & 100 \\
$N=100000$ & 28 & 32 & 100 & 83 & 99 & 100 \\
$N=200000$ & 45 & 52 & 100 & 94 & 100 & 100 \\
\hline
\end{tabular}

\subsection{Flexural beam}

Finally, a flexural beam structure is considered to validate the presented damage detection tests for damages of varying location, severity and spatial extent. To approach more realistic engineering tasks, a more complex model with a considerably higher number of DOFs is introduced. The model comprises 40 equally sized finite $2 \mathrm{D}$ elastic beam elements (Figure 8) allowing two translational and one rotational displacements at each node. The beam has a fixed support on one end and a movable support on the other end, so the system is statically indeterminate and four of the altogether 123 DOFs of the model are restricted. The beam has invariable cross-sectional and material properties in the undamaged state. Damage is modeled by a successive reduction of Young's modulus in selected finite elements, which has a linear impact on the stiffness at the affected elements. Thereby, damage scenarios with stiffness reductions of $20 \%$ to $50 \%$ in steps of $10 \%$ have been realized for four different locations of a 1-element sized damage and two 
different locations of a 2-element sized damage along the beam. Note that the 1-element damages with $20 \%$ stiffness reduction lead to less than $0.6 \%$ reduction of the natural frequencies and thus have a much smaller impact on the beam than the damage of the same magnitude in the previous truss example, which is due to the higher complexity and size of the beam model.

For each structural state, 200 data sets with each $N=100000$ acceleration samples at the six sensor positions were generated at a sampling frequency of $500 \mathrm{~Hz}$ from white noise excitation with a random covariance matrix $Q$ as in the truss example. $5 \%$ white noise were added on the generated outputs.

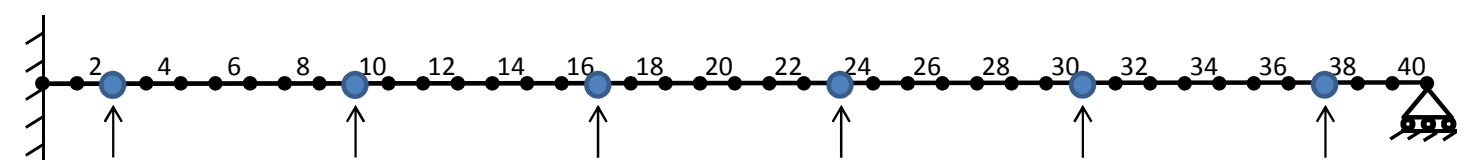

Figure 8: Flexural beam with element numbers and six sensors.

The damage detection tests (a)-(c) were computed on each of the data sets for the six different damage scenarios. The power of the test was evaluated for a 5\% type I error in the reference state. The results are summarized in Table 2 where it can be seen that the new damage detection test (c) clearly outperforms the conventional tests for all considered damage scenarios under changing excitation covariance. Damages of moderate magnitude can be detected at all considered positions of the beam with the new test, reaching $100 \%$ power of the test, while this is not possible with the conventional tests. Note that the acceptable power of the test for real world applications depends entirely on the application domain, the false alarms acceptance from the user and the minimal damage extent that one wishes to detect.

Table 2: Power of the damage detection tests (in \%) for different stiffness reductions at different beam elements and 5\% type I error.

\begin{tabular}{|c|c|c|c|c|c|c|c|c|c|c|c|c|c|c|c|c|c|c|}
\hline & \multicolumn{3}{|c|}{ element 3} & \multicolumn{3}{|c|}{ element 11} & \multicolumn{3}{|c|}{ element 21} & \multicolumn{3}{|c|}{ element 34} & \multicolumn{3}{|c|}{ elements $10+11$} & \multicolumn{3}{|c|}{ elements $22+23$} \\
\hline & (a) & (b) & (c) & (a) & (b) & (c) & (a) & (b) & (c) & (a) & (b) & (c) & (a) & (b) & (c) & (a) & (b) & (c) \\
\hline $20 \%$ & 9 & 8 & 19 & 8 & 11 & 23 & 7 & 6 & 9 & 13 & 12 & 27 & 15 & 24 & 95 & 18 & 25 & 87 \\
\hline $30 \%$ & 11 & 18 & 66 & 12 & 17 & 79 & 11 & 11 & 27 & 15 & 26 & 91 & 47 & 60 & 100 & 41 & 68 & 100 \\
\hline $40 \%$ & 36 & 34 & 100 & 30 & 36 & 100 & 20 & 26 & 88 & 35 & 52 & 100 & 66 & 100 & 100 & 70 & 98 & 100 \\
\hline $50 \%$ & 55 & 74 & 100 & 56 & 78 & 100 & 32 & 41 & 100 & 60 & 89 & 100 & 89 & 100 & 100 & 86 & 100 & 100 \\
\hline
\end{tabular}

\section{Conclusions}

In this paper, a damage detection test robust to changes in the ambient excitation properties has been derived. This test builds upon the statistical framework proposed in [12] and the residual proposed in [15]. A precise estimate of the covariance of the robust residual has been derived and it has been shown that the residual enjoys the property of having a constant covariance even in the context of varying excitation properties. Also, a numerically robust computation of the damage detection test was proposed, leading to a numerically and statistically robust damage detection algorithm, unlike previous endeavors. The method has been validated on simulated data, where its effectiveness was shown. A higher contrast of the damage index values between the reference and damage states was achieved, and the empirical distributions are better separated and more concentrated (narrower) allowing a clear damage detection decision. Finally, it has been shown that the new damage detection procedure is also more sensitive under lower sample length than previously proposed approaches in the same framework. These two advantages show that this new damage detection test is better suited for being embedded in an automated structural health monitoring system. 


\section{Acknowledgment}

The support from the NSF under the Hazard Mitigation and Structural Engineering Program Grant 1000391 for the first author and from the European project FP7-PEOPLE-2009-IAPP 251515 ISMS for the second author is gratefully acknowledged.

\section{Appendix A. Residual covariance and sensitivity computation for residual $\zeta$}

\section{Appendix A.1. Estimation of the residual covariance}

The residual covariance $\Sigma_{\zeta}$ depends on the covariance of the vectorized Hankel matrix

$$
\Sigma_{\mathcal{H}} \stackrel{\text { def }}{=} \lim _{N \rightarrow \infty} \operatorname{cov}\left(\sqrt{N} \operatorname{vec}\left(\widehat{\mathcal{H}}_{p+1, q}\right)\right)
$$

where $\widehat{\mathcal{H}}_{p+1, q}$ is computed on $N$ data samples. Then, the asymptotic residual covariance follows from the residual definitions in (6) or (14) as

$$
\Sigma_{\zeta}=\left(I \otimes S^{T}\right) \Sigma_{\mathcal{H}}(I \otimes S),
$$

where $S=S\left(\theta_{0}\right)$ for the parametric residual (6) and $S=\bar{S}$ for the non-parametric residual (14). An estimate of the residual covariance follows as

$$
\widehat{\Sigma}_{\zeta}=\left(I \otimes S^{T}\right) \widehat{\Sigma}_{\mathcal{H}}(I \otimes S),
$$

where the estimate $\widehat{\Sigma}_{\mathcal{H}}$ is obtained from a data sample $\left(y_{k}\right)_{k=1, \ldots, N}$ as follows. The available data is separated into $n_{b}$ blocks having the same length $N_{b}$ for simplicity, with $n_{b} \cdot N_{b}=N$. Each block may be long enough to assume statistical independence between the blocks. The correlations and the corresponding Hankel matrix

$$
\widehat{R}_{i}^{(j)}=\frac{1}{N_{b}} \sum_{k=1+(j-1) N_{b}}^{j N_{b}} y_{k} y_{k-i}^{T}, \quad \widehat{\mathcal{H}}_{p+1, q}^{(j)}=\operatorname{Hank}\left(\widehat{R}_{i}^{(j)}\right)
$$

are computed for each of the blocks. Then, $\widehat{\mathcal{H}}_{p+1, q}=\frac{1}{n_{b}} \sum_{j=1}^{n_{b}} \widehat{\mathcal{H}}_{p+1, q}^{(j)}$ and the covariance estimate of the vectorized Hankel matrix follows from the covariance of the sample mean as

$$
\widehat{\Sigma}_{\mathcal{H}}=\frac{N_{b}}{n_{b}-1} \sum_{j=1}^{n_{b}} \operatorname{vec}\left(\widehat{\mathcal{H}}_{p+1, q}^{(j)}-\widehat{\mathcal{H}}_{p+1, q}\right) \operatorname{vec}\left(\widehat{\mathcal{H}}_{p+1, q}^{(j)}-\widehat{\mathcal{H}}_{p+1, q}\right)^{T} .
$$

\section{Appendix A.2. Residual sensitivity}

The asymptotic residual sensitivity $\mathcal{J}_{\zeta}$ was derived in $[12,13]$ and yields

$$
\mathcal{J}_{\zeta}=\left(O_{p+1}\left(\theta_{0}\right)^{\dagger} \mathcal{H}_{p+1, q} \otimes S\left(\theta_{0}\right)\right)^{T} O_{p+1}^{\prime}\left(\theta_{0}\right)
$$

where $O_{p+1}\left(\theta_{0}\right)$ is the parametric observability matrix and $O_{p+1}^{\prime}\left(\theta_{0}\right)$ is the derivative of the vectorized parametric observability matrix with respect to $\theta_{0}$. Both matrices are obtained as follows. Based on the fact that all modes appear in conjugated complex pairs in structural vibration analysis, the system parameter $\theta_{0}$ defined in (2) can be separated into conjugated complex pairs such that

$$
\theta_{0}=\left[\begin{array}{l}
\theta_{\mathrm{c}} \\
\theta_{\mathrm{c}}^{*}
\end{array}\right]
$$

where * denotes the complex conjugate. In the same way, $\Phi_{\mathrm{c}}$ and $\Delta_{\mathrm{c}} \stackrel{\text { def }}{=} \operatorname{diag}\left(\Lambda_{\mathrm{c}}\right)$ are defined such that $\Phi=\left[\Phi_{\mathrm{c}} \quad \Phi_{\mathrm{c}}^{*}\right]$ and $\Lambda=\left[\begin{array}{ll}\Lambda_{\mathrm{c}}^{T} & \Lambda_{\mathrm{c}}^{* T}\end{array}\right]^{T}$. Then, define the (real-valued) parametric observability matrix as

$$
O_{p+1}\left(\theta_{0}\right) \stackrel{\operatorname{def}}{=}\left[\mathfrak{R}\left(\widetilde{O}_{p+1}\left(\theta_{\mathrm{c}}\right)\right) \quad \mathfrak{J}\left(\widetilde{O}_{p+1}\left(\theta_{\mathrm{c}}\right)\right)\right]=\left[\begin{array}{cc}
\mathfrak{R}\left(\Phi_{\mathrm{c}}\right) & \mathfrak{J}\left(\Phi_{\mathrm{c}}\right) \\
\mathfrak{R}\left(\Phi_{\mathrm{c}} \Delta_{\mathrm{c}}\right) & \mathfrak{J}\left(\Phi_{\mathrm{c}} \Delta_{\mathrm{c}}\right) \\
\vdots & \vdots \\
\mathfrak{R}\left(\Phi_{\mathrm{c}} \Delta_{\mathrm{c}}^{p}\right) & \mathfrak{J}\left(\Phi_{\mathrm{c}} \Delta_{\mathrm{c}}^{p}\right)
\end{array}\right],
$$


where

$$
\widetilde{O}_{p+1}\left(\theta_{\mathrm{c}}\right) \stackrel{\text { def }}{=}\left[\begin{array}{c}
\Phi_{\mathrm{c}} \\
\Phi_{\mathrm{c}} \Delta_{\mathrm{c}} \\
\vdots \\
\Phi_{\mathrm{c}} \Delta_{\mathrm{c}}^{p}
\end{array}\right]
$$

is the complex-valued observability matrix containing only half of the modes and $\mathfrak{R}(\cdot)$ and $\mathfrak{J}(\cdot)$ denote the real and imaginary part. Define

$$
\Lambda_{i}^{(p) \stackrel{\text { def }}{=}}\left[\begin{array}{lllll}
1 & \lambda_{i} & \lambda_{i}^{2} & \ldots & \lambda_{i}^{p}
\end{array}\right]^{T}, \Lambda_{i}^{\prime(p)} \stackrel{\text { def }}{=}\left[\begin{array}{lllll}
0 & 1 & 2 \lambda_{i} & \ldots & p \lambda_{i}^{p-1}
\end{array}\right]^{T}
$$

for $1 \leq i \leq m$, where $m$ is the number of conjugated complex mode pairs. Then the complex-valued derivative of the vectorized observability matrix $\widetilde{O}_{p+1}\left(\theta_{\mathrm{c}}\right)$ writes

$$
\left.{\widetilde{O_{p+1}^{\prime}}}_{p}^{\prime}\left(\theta_{\mathrm{c}}\right) \stackrel{\operatorname{def}}{=} \frac{\partial \operatorname{vec}\left(\widetilde{O}_{p+1}(\tilde{\theta})\right)}{\partial \tilde{\theta}}\right|_{\tilde{\theta}=\theta_{\mathrm{c}}}=\left[\begin{array}{ccc|ccc}
\Lambda_{1}^{\prime(p)} \otimes \varphi_{1} & & 0 & \Lambda_{1}^{(p)} \otimes I_{r} & & 0 \\
& \ddots & & \ddots & \\
0 & & \Lambda_{m}^{\prime(p)} \otimes \varphi_{m} & 0 & & \Lambda_{m}^{(p)} \otimes I_{r}
\end{array}\right],
$$

and the derivative of the real-valued parametric observability matrix $O_{p+1}^{\prime}\left(\theta_{0}\right)$ for the residual sensitivity in (A.3) yields

$$
O_{p+1}^{\prime}\left(\theta_{0}\right)=\left[\begin{array}{cc}
\mathfrak{R}\left({\widetilde{O^{\prime}}}_{p+1}^{\prime}\left(\theta_{\mathrm{c}}\right)\right) & -\mathfrak{J}\left({\widetilde{O_{p+1}^{\prime}}}_{p+1}^{\prime}\left(\theta_{\mathrm{c}}\right)\right) \\
\mathfrak{J}\left({\widetilde{O_{p+1}^{\prime}}}_{p+1}^{\prime}\left(\theta_{\mathrm{c}}\right)\right) & \mathfrak{R}\left({\widetilde{O_{p+1}^{\prime}}}_{p+1}^{\prime}\left(\theta_{\mathrm{c}}\right)\right)
\end{array}\right] .
$$

A consistent estimate $\widehat{\mathcal{J}}_{\zeta}$ is obtained by replacing $\mathcal{H}_{p+1, q}$ by $\widehat{\mathcal{H}}_{p+1, q}$.

\section{Appendix B. Residual covariance and sensitivity computation for new residual $\xi$}

\section{Appendix B.1. Covariance}

The covariance of the robust residual depends on the covariance of the singular vectors of the Hankel matrix in the reference state. The covariance estimate $\widehat{\Sigma}_{\mathcal{H}}$ of the vectorized Hankel matrix itself was already obtained in Appendix A.1. Thus, a possibility to compute the estimate $\widehat{\Sigma}_{\xi}$ is to propagate the covariance of the vectorized Hankel matrix to the covariance of the singular vectors by a sensitivity analysis [22]. Then it holds

$$
\operatorname{cov}\left(\sqrt{N} \operatorname{vec} \widehat{U}_{1}\right)=\mathcal{J}_{\widehat{U}_{1}} \widehat{\Sigma}_{\mathcal{H}} \mathcal{J}_{\widehat{U}_{1}}^{T},
$$

where $\mathcal{J}_{\widehat{U}_{1}}$ is the sensitivity of the left singular vectors $\operatorname{vec}\left(\widehat{U}_{1}\right)$ with respect to $\operatorname{vec}\left(\widehat{\mathcal{H}}_{p+1, q}\right)$, and it follows

$$
\widehat{\Sigma}_{\xi}=\left(I \otimes S^{T}\right) \mathcal{J}_{\widehat{U}_{1}} \widehat{\Sigma}_{\mathcal{H}} \mathcal{J}_{\widehat{U}_{1}}^{T}(I \otimes S) .
$$

The computation of $\mathcal{J}_{\widehat{U}_{1}}$ was derived in [22] and is numerically costly. A more efficient computation of the required singular vector sensitivities is summarized in the following proposition.

Proposition 1. Let the SVD of $\widehat{\mathcal{H}}_{p+1, q}$ in (11) with singular vectors and values

$$
\widehat{U}_{1}=\left[\begin{array}{lll}
u_{1} & \ldots & u_{n}
\end{array}\right], \widehat{V}_{1}=\left[\begin{array}{lll}
v_{1} & \ldots & v_{n}
\end{array}\right], \widehat{\Delta}_{1}=\operatorname{diag}\left\{\sigma_{1}, \ldots, \sigma_{n}\right\}
$$

be given. For $j=1, \ldots, n$ define

$$
\begin{aligned}
& K_{j} \stackrel{\text { def }}{=} \frac{\widehat{\mathcal{H}}_{p+1, q}}{\sigma_{j}}\left(I+\left[\begin{array}{c}
0 \\
2 v_{j}^{T}
\end{array}\right]-\frac{\widehat{\mathcal{H}}_{p+1, q}^{T} \widehat{\mathcal{H}}_{p+1, q}}{\sigma_{j}^{2}}\right)^{-1}, \\
& E_{j} \stackrel{\text { def }}{=}\left[I+K_{j}\left(\frac{\widehat{\mathcal{H}}_{p+1, q}^{T}}{\sigma_{j}}-\left[\begin{array}{c}
0 \\
u_{j}^{T}
\end{array}\right]\right) \quad K_{j}\right], \quad F_{j} \stackrel{\text { def }}{=} \frac{1}{\sigma_{j}}\left[\begin{array}{l}
v_{j}^{T} \otimes\left(I-u_{j} u_{j}^{T}\right) \\
\left(I-v_{j} v_{j}^{T}\right) \otimes u_{j}^{T}
\end{array}\right] .
\end{aligned}
$$


Then the sensitivity of the left singular vectors of $\widehat{\mathcal{H}}_{p+1, q}$ writes

$$
\mathcal{J}_{\widehat{U}_{1}}=\left[\begin{array}{c}
E_{1} F_{1} \\
\vdots \\
E_{n} F_{n}
\end{array}\right] .
$$

Proof. A detailed proof can be found in $[1,23]$.

\section{Appendix B.2. Sensitivity}

The computation of the sensitivity $\mathcal{J}_{\xi}$ is analogous to the computation of the sensitivity $\mathcal{J}_{\zeta}$ of $\zeta_{N}$ in (A.3), where $\mathcal{H}_{p+1, q}$ is replaced by $U_{1}$ and it follows

$$
\mathcal{J}_{\xi}=\left(O_{p+1}\left(\theta_{0}\right)^{\dagger} U_{1} \otimes S\left(\theta_{0}\right)\right)^{T} O_{p+1}^{\prime}\left(\theta_{0}\right),
$$

where ${ }^{\dagger}$ denotes the pseudoinverse and $\otimes$ denotes the Kronecker product. A consistent estimate $\widehat{\mathcal{J}}_{\xi}$ is obtained by replacing $U_{1}$ by $\widehat{U}_{1}$.

\section{Appendix C. Numerical considerations}

It is the objective to compute the tests (19) and (20), or equivalently the tests for a rank deficient covariance estimate

$$
\chi^{2}=\Upsilon^{T} \widehat{\Sigma}^{\dagger} \widehat{\mathcal{J}}\left(\widehat{\mathcal{J}}^{T} \widehat{\Sigma}^{\dagger} \widehat{\mathcal{J}}\right)^{\dagger} \widehat{\mathcal{J}}^{T} \widehat{\Sigma}^{\dagger} \Upsilon
$$

and

$$
\chi^{2}=\Upsilon^{T} \widehat{\Sigma}^{\dagger} \Upsilon
$$

in a numerically stable way. First, an efficient computation of the square root inverse of the covariance matrix estimate is presented, which takes a key role in the computation of the $\chi^{2}$-tests. Second, results from [21] for a numerically stable computation of the $\chi^{2}$-test are extended and discussed. Let $c=\operatorname{dim}(\theta)$ be the dimension of the underlying system parameter and $d=\operatorname{dim}(\Upsilon)$ the dimension of residual vector, such that $\widehat{\mathcal{J}} \in \mathbb{R}^{d \times c}$ and $\widehat{\Sigma} \in \mathbb{R}^{d \times d}$.

\section{Appendix C.1. Computation of square root inverse of $\widehat{\Sigma}$}

Denote $\widehat{\Sigma}^{-1 / 2} \in \mathbb{R}^{e x d}$ as a square root (pseudo-)inverse of the covariance matrix $\widehat{\Sigma}$ as defined in (21) for both the inverse and pseudoinverse of $\widehat{\Sigma}$. Note that in the full rank case, the dimensions of $\widehat{\Sigma}^{-1 / 2}$ yield $e \geq d$. In the rank deficient case, only $e \geq \operatorname{rank}(\overrightarrow{\bar{\Sigma}})$ holds and thus $e<d$ is possible. The square root inverse $\widehat{\Sigma}^{-1 / 2}$ is needed for a numerically stable computation of the $\chi^{2}$-test in the subsequent section and can be obtained efficiently as follows. For all considered damage detection tests, the respective covariance matrix estimates in (A.1) or (B.1) can be factorized into

$$
\widehat{\Sigma}=\mathcal{A} \widehat{\Sigma}_{\mathcal{H}} \mathcal{A}^{T},
$$

where $\mathcal{A}=\left(I \otimes S^{T}\right)$ or $\mathcal{A}=\left(I \otimes S^{T}\right) \mathcal{J}_{\widehat{U}_{1}}$, respectively, and the covariance estimate $\widehat{\Sigma}_{\mathcal{H}}$ of the vectorized Hankel matrix is obtained in (A.2). The latter matrix is obtained from cutting the sensor data into $n_{b}$ statistically independent blocks, on which instances of the Hankel matrix $\widehat{\mathcal{H}}_{p+1, q}^{(j)}$ are computed. Define

$$
\mathcal{K} \stackrel{\text { def }}{=} \sqrt{\frac{N_{b}}{n_{b}-1}}\left[\begin{array}{llll}
h_{1} & h_{2} & \ldots & h_{n_{b}}
\end{array}\right] \text { with } h_{j} \stackrel{\text { def }}{=} \operatorname{vec}\left(\widehat{\mathcal{H}}_{p+1, q}^{(j)}-\widehat{\mathcal{H}}_{p+1, q}\right)
$$

and the factorization property

$$
\widehat{\Sigma}_{\mathcal{H}}=\mathcal{K} \mathcal{K}^{T}
$$

follows from (A.2). Plugging this result into (C.3) yields

$$
\widehat{\Sigma}=(\mathcal{A K})(\mathcal{A K})^{T}
$$


and a square root (pseudo-)inverse of the covariance estimate $\widehat{\Sigma}$ can be written as

$$
\widehat{\Sigma}^{-1 / 2}=(\mathcal{A K})^{\dagger} .
$$

This relation provides an efficient way to compute the square root (pseudo-)inverse $\widehat{\Sigma}^{-1 / 2}$ of the covariance matrix $\widehat{\Sigma}$ in case of few available data blocks $n_{b}$, while completely avoiding the explicit computation of $\widehat{\Sigma}$. Instead, the matrix $\mathcal{K}$ (having $n_{b}$ columns) is directly filled with the estimates on the data blocks without further computations in (C.4). Then, in (C.5) the pseudoinverse of matrix $\mathcal{A K} \in \mathbb{R}^{d \times n_{b}}$ is computed and thus the matrix $\widehat{\Sigma}^{-1 / 2}$ has $e=n_{b}$ rows. If $n_{b}<d=\operatorname{dim} \Upsilon$, the computation of $\widehat{\Sigma}^{-1 / 2}$ in (C.5) is less costly than computing $\widehat{\Sigma}^{-1 / 2}$ directly from $\widehat{\Sigma} \in \mathbb{R}^{d \times d}$.

Moreover, the computation of the (pseudo-)inverse of matrix $\mathcal{A K}$ is numerically more stable than the square root (pseudo-)inverse of the squared matrix $\widehat{\Sigma}=(\mathcal{A K})(\mathcal{A K})^{T}$. Hence, using (C.5) may be favorable, even if $n_{b} \geq d$.

\section{Appendix C.2. Numerically stable computation of $\chi^{2}$-test}

In (22) and (24) numerically robust expressions for the computation of the $\chi^{2}$-tests were given, which hold both for the tests as stated in (19) and (20) and for the tests in rank deficient case in (C.1) and (C.2), respectively. We outline their origins and give some comments on conditions for these expressions.

Expression (22) for the non-parametric test follows directly from (21) and provides robustness by only using a matrix-vector product with $\widehat{\Sigma}^{-1 / 2}$.

Expression (24) was proposed in [21] and uses the fact that if the product

$$
\widehat{\Sigma}^{-1 / 2} \widehat{\mathcal{J}} \text { is full column rank, }
$$

then the thin QR decomposition

$$
\widehat{\Sigma}^{-1 / 2} \widehat{\mathcal{J}}=Q \mathcal{R}
$$

yields a square invertible upper triangular matrix $\mathcal{R}$ and matrix $Q$ having orthonormal columns with $Q^{T} Q=I$. Then, from (19) or (C.1) follows with the definition of $\widehat{\Sigma}^{-1 / 2}$ in (21)

$$
\begin{aligned}
\chi^{2} & =\Upsilon^{T}\left(\widehat{\Sigma}^{-1 / 2}\right)^{T} \widehat{\Sigma}^{-1 / 2} \widehat{\mathcal{J}}\left(\widehat{\mathcal{J}}^{T}\left(\widehat{\Sigma}^{-1 / 2}\right)^{T} \widehat{\Sigma}^{-1 / 2} \widehat{\mathcal{J}}\right)^{\dagger} \widehat{\mathcal{J}}^{T}\left(\widehat{\Sigma}^{-1 / 2}\right)^{T} \widehat{\Sigma}^{-1 / 2} \Upsilon \\
& =\Upsilon^{T}\left(\widehat{\Sigma}^{-1 / 2}\right)^{T} Q \mathcal{R}\left(\mathcal{R}^{T} Q^{T} Q \mathcal{R}\right)^{\dagger} \mathcal{R}^{T} Q^{T} \widehat{\Sigma}^{-1 / 2} \Upsilon \\
& =\Upsilon^{T}\left(\widehat{\Sigma}^{-1 / 2}\right)^{T} Q Q^{T} \widehat{\Sigma}^{-1 / 2} \Upsilon
\end{aligned}
$$

and finally expression (24). In this computation, no further matrix inversions and only numerical stable computations are used, once $\widehat{\Sigma}^{-1 / 2}$ is obtained. Note that condition (C.6) is required for the use of the sensitivity matrix $\widehat{\mathcal{J}}$ in the $\chi^{2}$-test. In practice, however, it can happen that condition (C.6) is not satisfied. More precisely, if the number of columns $c$ of matrix $\widehat{\mathcal{J}}(c=\operatorname{dim}(\theta))$ yields

$$
c \geq \operatorname{rank}\left(\widehat{\Sigma}^{-1 / 2} \widehat{\mathcal{J}}\right),
$$

condition (C.6) is violated, and $\widehat{\mathcal{J}}$ cancels out in the parametric $\chi^{2}$-test, leading to its non-parametric version: if condition (C.7) if fulfilled, a rank-revealing QR decomposition with column pivoting [24] of $\widehat{\mathcal{J}}^{T}\left(\widehat{\Sigma}^{-1 / 2}\right)^{T}=Q \mathcal{R} \Pi^{T}$ is possible, where $\mathcal{R}$ is invertible, $Q^{T} Q=I$ and $\Pi$ is a permutation matrix with $\Pi^{T} \Pi=\Pi \Pi^{T}=I$. Plugging this $\mathrm{QR}$ decomposition into (C.1) yields

$$
\begin{aligned}
\chi^{2} & =\Upsilon^{T}\left(\widehat{\Sigma}^{-1 / 2}\right)^{T} \Pi \mathcal{R}^{T} Q^{T}\left(Q \mathcal{R} \Pi^{T} \Pi \mathcal{R}^{T} Q^{T}\right)^{\dagger} Q \mathcal{R} \Pi^{T} \widehat{\Sigma}^{-1 / 2} \Upsilon \\
& =\Upsilon^{T} \widehat{\Sigma}^{-1} \Upsilon,
\end{aligned}
$$

because $\left(Q \mathcal{R} \mathcal{R}^{T} Q^{T}\right)^{\dagger}=Q \mathcal{R}^{-T} \mathcal{R}^{-1} Q^{T}$ as $Q$ has orthonormal columns and $\mathcal{R}$ is invertible. Thus, the parametric $\chi^{2}$-test boils down to its non-parametric version, if condition (C.7) is fulfilled. This is for example the case, when the number of parameters in $\theta$ is bigger than the dimension of the residual. Also, when the number of parameters in $\theta$ is bigger than the number of data blocks $n_{b}$ for the covariance computation, condition (C.7) is fulfilled due to (C.5). In these cases, the residual's sensitivity will disappear in the $\chi^{2}$-test statistics (C.1). Hence, the number of data blocks must satisfy $n_{b}>c$ as a necessary condition for the use of a sensitivity matrix $\mathcal{J}$. Otherwise, the non-parametric test can be applied directly. 


\section{References}

[1] M. Döhler, L. Mevel, Robust subspace based fault detection, in: Proc. 18th IFAC World Congress, Milan, Italy, 2011.

[2] C. Farrar, S. Doebling, D. Nix, Vibration-based structural damage identification, Philosophical Transactions of the Royal Society A: Mathematical, Physical and Engineering Science 359 (1778) (2001) 131-149.

[3] S. Doebling, C. Farrar, M. Prime, A summary review of vibration-based damage identification methods, Shock and Vibration Digest 30 (2) (1998) 91-105.

[4] E. Carden, P. Fanning, Vibration based condition monitoring: a review, Structural Health Monitoring 3 (4) (2004) $355-377$.

[5] K. Worden, C. Farrar, G. Manson, G. Park, The fundamental axioms of structural health monitoring, Proceedings of the Royal Society A: Mathematical, Physical and Engineering Science 463 (2082) (2007) 1639-1664.

[6] J. Kullaa, Damage detection of the Z24 Bridge using control charts, Mechanical Systems and Signal Processing 17 (1) (2003) $163-170$.

[7] F. Magalhães, A. Cunha, E. Caetano, Dynamic monitoring of a long span arch bridge, Engineering Structures 30 (11) (2008) $3034-3044$.

[8] L. Ramos, L. Marques, P. Lourenço, G. De Roeck, A. Campos-Costa, J. Roque, Monitoring historical masonry structures with operational modal analysis: Two case studies, Mechanical Systems and Signal Processing 24 (5) (2010) 1291-1305, special Issue: Operational Modal Analysis.

[9] K. Worden, G. Manson, N. Fieller, Damage detection using outlier analysis, Journal of Sound and Vibration 229 (3) (2000) $647-667$.

[10] A. Yan, P. De Boe, J. Golinval, Structural damage diagnosis by Kalman model based on stochastic subspace identification, Structural Health Monitoring 3 (2) (2004) 103-119.

[11] D. Bernal, Kalman filter damage detection in the presence of changing process and measurement noise, Mechanical Systems and Signal Processing. In press. doi:10.1016/j.ymssp.2013.02.012.

[12] M. Basseville, M. Abdelghani, A. Benveniste, Subspace-based fault detection algorithms for vibration monitoring, Automatica 36 (1) (2000) 101-109.

[13] M. Basseville, L. Mevel, M. Goursat, Statistical model-based damage detection and localization: subspace-based residuals and damage-tonoise sensitivity ratios, Journal of Sound and Vibration 275 (3) (2004) 769-794.

[14] E. Balmès, M. Basseville, F. Bourquin, L. Mevel, H. Nasser, F. Treyssède, Merging sensor data from multiple temperature scenarios for vibration-based monitoring of civil structures, Structural Health Monitoring 7 (2) (2008) 129-142.

[15] A.-M. Yan, J.-C. Golinval, Null subspace-based damage detection of structures using vibration measurements, Mechanical Systems and Signal Processing 20 (3) (2006) 611-626.

[16] P. Van Overschee, B. De Moor, Subspace Identification for Linear Systems: Theory, Implementation, Applications, Kluwer, 1996.

[17] A. Benveniste, M. Basseville, G. Moustakides, The asymptotic local approach to change detection and model validation, IEEE Transactions on Automatic Control 32 (7) (1987) 583-592.

[18] G. Moustakides, A. Benveniste, Detecting changes in the AR parameters of a nonstationary ARMA process, Stochastics - An International Journal of Probability and Stochastic Processes 16 (1) (1986) 137-155.

[19] E. Bura, R. Pfeiffer, On the distribution of the left singular vectors of a random matrix and its applications, Statistics \& Probability Letters 78 (15) (2008) 2275-2280.

[20] C. Rao, S. Mitra, Generalized inverse of a matrix and its applications, J. Wiley, New York, 1971.

[21] Q. Zhang, M. Basseville, Advanced numerical computation of $\chi^{2}$-tests for fault detection and isolation, in: 5th Symp. Fault Detection, Supervision and Safety for Technical Processes (SAFEPROCESS), Washington, USA, 2003, pp. 211-216.

[22] R. Pintelon, P. Guillaume, J. Schoukens, Uncertainty calculation in (operational) modal analysis, Mechanical Systems and Signal Processing 21 (6) (2007) 2359-2373.

[23] M. Döhler, L. Mevel, Efficient multi-order uncertainty computation for stochastic subspace identification, Mechanical Systems and Signal Processing 38 (2) (2013) 346-366.

[24] G. Golub, C. Van Loan, Matrix computations, 3rd Edition, Johns Hopkins University Press, 1996. 